Research Paper

\title{
Hyaluronan activates Hyal-2/WWOX/Smad4 signaling and causes bubbling cell death when the signaling complex is overexpressed
}

\author{
Li-Jin Hsu ${ }^{1,3, *}$, Qunying Hong ${ }^{1, *}$, Shur-Tzu Chen ${ }^{1,4, *}$, Hsiang-Lin Kuo ${ }^{2, *}$, Lori Schultz ${ }^{1}$, \\ John Heath ${ }^{1}$, Sing-Ru Lin ${ }^{2}$, Ming-Hui Lee ${ }^{2}$, Dong-Zhang Lí ${ }^{2}$, Zih-Ling Lí2, Hui-Ching \\ Cheng $^{3}$, Gerard Armand7, Nan-Shan Chang ${ }^{1,2,5,6}$ \\ ${ }^{1}$ Guthrie Research Institute, Laboratory of Molecular Immunology, Sayre, PA, USA \\ ${ }^{2}$ Institute of Molecular Medicine, National Cheng Kung University College of Medicine, Tainan, Taiwan, ROC \\ ${ }^{3}$ Department of Medical Laboratory Science and Biotechnology, National Cheng Kung University College of Medicine, Tainan, \\ Taiwan, ROC \\ ${ }^{4}$ Department of Cell Biology and Anatomy, National Cheng Kung University College of Medicine, Tainan, Taiwan, ROC \\ ${ }^{5}$ Advanced Optoelectronic Technology Center, National Cheng Kung University, Tainan, Taiwan, ROC \\ ${ }^{6}$ Graduate Institute of Biomedical Sciences, College of Medicine, China Medical University, Taichung, Taiwan, ROC \\ ${ }^{7}$ Glycomed Research Inc., Hastings on Hudson, New York, NY, USA \\ *Equal contributions among these authors
}

Correspondence to: Nan-Shan Chang, email: nschang13827@gmail.com

Keywords: hyaluronan, hyaluronidase, Hyal-2, Smad, WWOX

Received: April 08, $2016 \quad$ Accepted: October 17, $2016 \quad$ Published: November 10, 2016

\section{ABSTRACT}

Malignant cancer cells frequently secrete significant amounts of transforming growth factor beta (TGF- $\beta$ ), hyaluronan (HA) and hyaluronidases to facilitate metastasizing to target organs. In a non-canonical signaling, TGF- $\beta$ binds membrane hyaluronidase Hyal-2 for recruiting tumor suppressors WWOX and Smad4, and the resulting Hyal-2/WWOX/Smad4 complex is accumulated in the nucleus to enhance SMAD-promoter dependent transcriptional activity. Yeast two-hybrid analysis showed that WWOX acts as a bridge to bind both Hyal-2 and Smad4. When WWOX-expressing cells were stimulated with high molecular weight $H A$, an increased formation of endogenous Hyal-2/WWOX/Smad4 complex occurred rapidly, followed by relocating to the nuclei in 20-40 min. In WWOX-deficient cells, HA failed to induce Smad2/3/4 relocation to the nucleus. To prove the signaling event, we designed a real time trimolecular FRET analysis and revealed that HA induces the signaling pathway from ectopic Smad4 to WWOX and finally to p53, as well as from Smad4 to Hyal-2 and then to WWOX. An increased binding of the Smad4/Hyal-2/WWOX complex occurs with time in the nucleus that leads to bubbling cell death. In contrast, HA increases the binding of Smad4/WWOX/p53, which causes membrane blebbing but without cell death. In traumatic brain injury-induced neuronal death, the Hyal-2/WWOX complex was accumulated in the apoptotic nuclei of neurons in the rat brains in $24 \mathrm{hr}$ post injury, as determined by immunoelectron microscopy. Together, HA activates the Hyal-2/WWOX/Smad4 signaling and causes bubbling cell death when the signaling complex is overexpressed.

\section{INTRODUCTION}

Hyaluronidases Hyal-1 and Hyal-2 and hyaluronan (HA) are associated with embryonic development, morphogenesis, wound healing, neurodegeneration, and cancer progression [1-4]. Degraded hyaluronan is critical for cancer cell-associated angiogenesis, survival and metastasis [1-4]. Hyal-1 is a lysosomal enzyme that is secreted from cells, and is considered as a tumor suppressor [5]. However, it enhances extravasation 
and metastasis of many types of cancer cells [6-8]. Hyal-2 is a lysosomal protein and a candidate tumor suppressor [9], and is also anchored on cell surface via glycosylphosphatidylinositol (GPI) [10]. Hyal2 participates in glycalyx formation [11]. Surface Hyal-2 is a co-receptor with CD44 for HA [1]. The enzymatic activity of Hyal-2 can be induced under acidic environment [12]. Hyaluronidase PH-20 is also a membrane GPI-anchored protein, and is released as a soluble form [1]. PH-20 is expressed in breast and other cancer tissues $[1,13]$. We determined that bovine testicular PH-20 increases the susceptibility of murine L929 fibroblasts and prostate LNCaP cancer cells to tumor necrosis factor (TNF or TNF- $\alpha$ ) cytotoxicity [14, 15]. PH-20 induces the expression of proapoptotic $\mathrm{p} 53$ and WW domain-containing oxidoreductase (WWOX, WOX1, or FOR) [16-19], which contributes in part to the increased TNF sensitivity in murine L929 fibroblasts.

HA signaling via CD44 receptor has been well documented $[1-4,20]$. In a non-canonical pathway, transforming growth factor beta (TGF- $\beta$ ) binds membrane Hyal-2 and then recruits tumor suppressors WWOX and Smad4, and the resulting Hyal-2/WWOX/Smad4 induces SMAD-dependent transcriptional activation in the nucleus $[18,21,22]$. Recently, membrane Hyal-2 is shown to bind a 31-amino-acid peptide Zfra (zinc finger-like protein that regulates apoptosis) for leading to the activation of spleen Hyal-2 ${ }^{+}$CD3- CD19- Z cell in memory anticancer response [23]. Hyaluronidases PH-20, Hyal-1 and Hyal-2 induce the expression of tumor suppressor WWOX [16, 17, 24]. Human $W W O X$ gene is located on a chromosomal fragile site $16 q 23[25,26]$. Loss of heterozygosity ( $\mathrm{LOH})$ and alterations of $W W O X$ gene have been shown in a variety of cancers [17, 18, 22, 27-31]. WWOX-mediated suppression of cancer cell growth has been established in Drosophila [32], in cell lines, and in drug-induced WWOX expression for cancer treatment [33]. Notably, null mutations of $W W O X / W$ wox gene in humans, rats and mice result in severe neural diseases (e.g. microcephaly, seizure, ataxia, etc.), growth retardation, metabolic disorders, and significantly shortening of life span [22, 34-36]. Essential no spontaneous cancer formation has been seen in the newborns [22, 34-36].

WWOX possesses two $N$-terminal WW domains (containing conserved tryptophan residues), a nuclear localization sequence (NLS), and a $C$-terminal shortchain alcohol dehydrogenase/reductase (ADH/SDR) domain [16-18, 22, 27-31]. WWOX undergoes Tyr33 phosphorylation, binds Ser46-phosphorlated p53, and translocates with p53 to the nucleus in response to stress responses, including anisomycin, TNF- $\alpha$ and UV irradiation [37-39], low energy constant light for photoreceptor degeneration [40], and 17 $\beta$-estradiol [41]. Both p53 and WWOX induce apoptosis synergistically [16, 37-39]. Calcium ionophore and phorbol ester induce WWOX to undergo Ser14 phosphorylation, which leads to terminal maturation of $\mathrm{T}$ cell acute lymphoblastic leukemia via I $\mathrm{B} \alpha / \mathrm{ERK} / \mathrm{WWOX}$ signaling [42].

A portion of cytosolic WWOX is anchored by Hyal-2 or Ezrin onto the cell membrane [21, 43]. Here, we determined that in WWOX-expressing cells, high molecular weight HA induced the formation of WWOX, Hyal-2 and Smads complex, and the complex underwent relocation to the nucleus. The HA-induced signaling of ectopic Smad/Hyal-2/WWOX was confirmed by real time tri-molecular FRET (Förster resonance energy transfer) microscopy [42]. The Smad/Hyal-2/WWOX complex relocated to the nucleus to induce cell death. Meanwhile, a signaling pathway of Smad/WWOX/ p53 was also activated but failed to cause cell death. Functional significance of the Hyal-2/WWOX signaling was tested in a traumatic brain injury model in rat, and the signaling revealed a critical role of nuclear Hyal-2 and WWOX in causing apoptosis under stress conditions.

\section{RESULTS}

\section{HA induction of nuclear accumulation of WWOX, ERK and smads in WWOX-expressing cells}

HA either enhances or blocks the TGF- $\beta 1$ signaling [24]. HA binds cell surface CD44 receptor, then activates the closely associated TGF-receptor type I (T $\beta R I)$, and leads to activation of Smads in triplenegative breast metastatic MDA-MB-231 cells [44]. These cells lack estrogen receptor (ER), progesterone receptor (PR) and Her2/neu [44]. Here, we determined that exposure of malignant prostate DU145 cells to high-molecular-weight hyaluronan $\left(10^{5}-10^{6} \mathrm{kDa}\right)$ for $1 \mathrm{hr}$ resulted in nuclear translocation of full-length WWOX (46 kDa), isoform WWOX2 (42 kDa), p-FAK, p-Smad2/3, Smad4, p-ERK, and pS46-p53 (Figure $1 \mathrm{~A} ; \sim 100-300 \%$ increased in nuclear accumulation). In contrast, pS15-p53 did not translocate to the nuclei. Ser46 phosphorylation is essential for p53 to mediate apoptosis [38, 39, 41]. In time-course experiments, HA, as low as $100 \mathrm{ng} / \mathrm{ml}$, caused nuclear accumulation of WWOX, Hyal-2 and Smad4 in 20 min post stimulation (Supplementary Figure S1). Similar results were observed using 25-100 $\mu \mathrm{g} / \mathrm{ml}$ HA (data not shown). DU145 cells do not express androgen receptors (AR) [45], but have an intact TGF- $\beta$ signaling pathway [46] and express mutant p53 [47]. Similarly, HA induced nuclear accumulation of WWOX, WWOX2, p53, p-ERK and p-Smad2/3 in WWOX-expressing breast MCF7 cells during treatment for $1 \mathrm{hr}$ (Figure 1B; 40$100 \%$ increased in nuclear accumulation). MCF7 cells express functional ER $[41,44]$ and wild type p53 and WWOX [41]. 
A

DU145

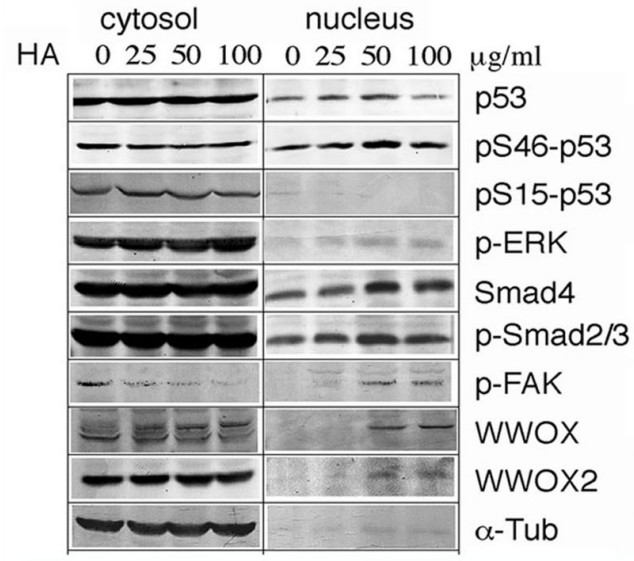

B HA $\frac{\text { cytosol }}{-+} \frac{\text { nucleus }}{-+}$

p53

Smad4

p-Smad2/3

WWOX

WWOX2

$\alpha$-Tub

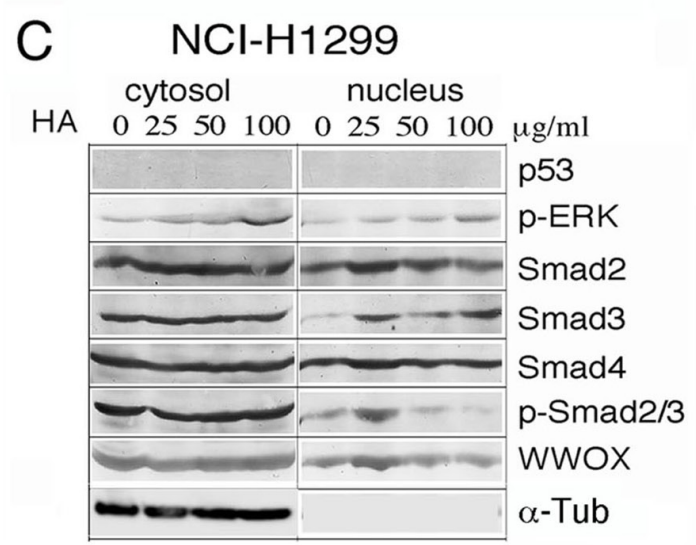

D

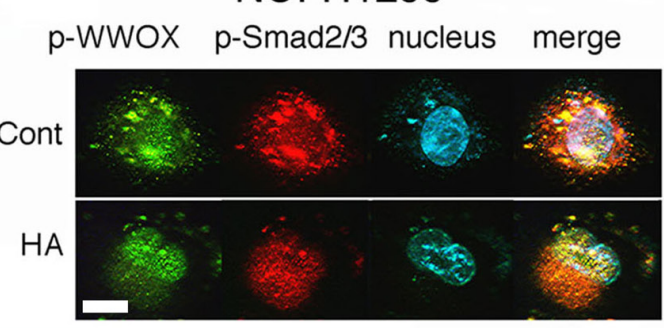

E

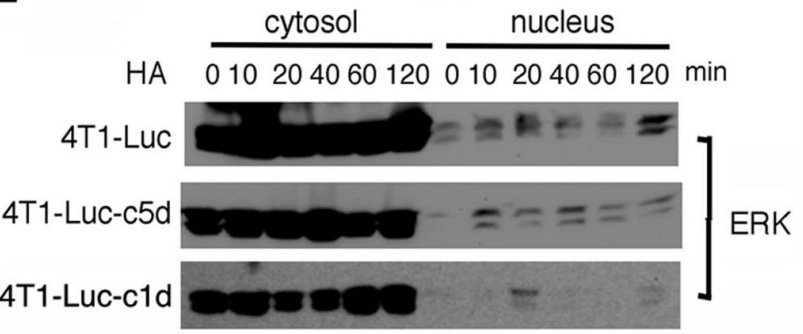

F THP-1

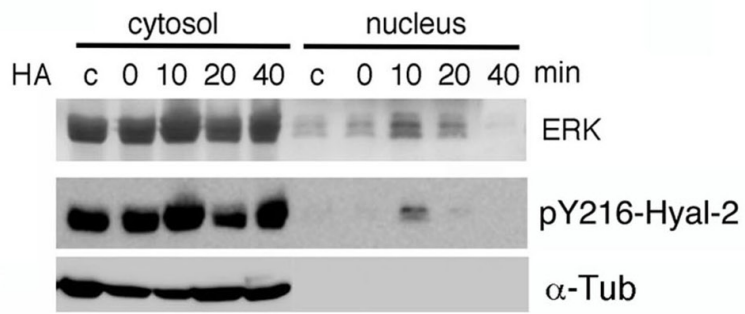

G Jurkat

HA C 01020406080120 c 01020406080120 min

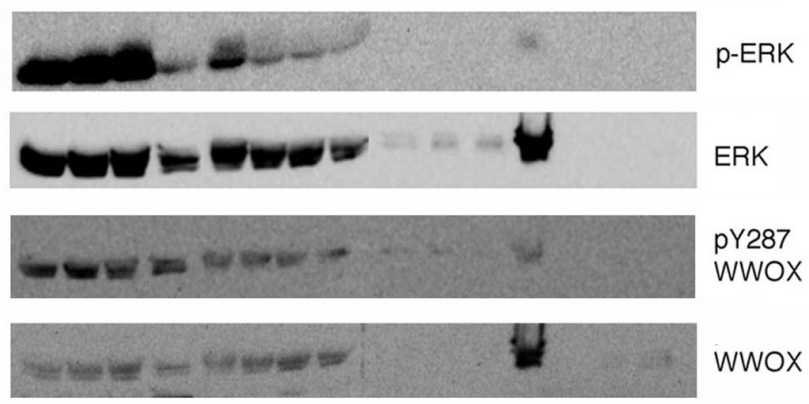

لש שصשص $\alpha$-Tub

Figure 1: Hyaluronan induces nuclear accumulation of WWOX, Smads and others proteins. A. Prostate DU145 cells were treated with high-molecular-weight hyaluronan (HA; $10^{5}-10^{6} \mathrm{kDa} ; 50 \mu \mathrm{g} / \mathrm{ml}$; Sigma) for $1 \mathrm{hr}$. Nuclear translocation of full length WWOX, isoform WWOX2, p-FAK, p-Smad2/3, Smad4, p-ERK, and pSer46-p53 is shown ( $100-300 \%$ increases). Time course analysis showed that in response to a low level of HA at $100 \mathrm{ng} / \mathrm{ml}$, WWOX and Smad4 relocated to the nucleus in 40 min (Supplementary Figure S1). B. Breast MCF7 cells were treated with HA for $1 \mathrm{hr}$. Nuclear translocation of WWOX, WWOX2, p53, p-ERK and p-Smad2/3 was shown. C, D. p53-deficient NCI-H1299 cells were treated with medical grade HA for 1 hr. Nuclear translocation of WWOX, p-WWOX (Tyr33 phosphorylation) and Smads is shown by Western blotting and fluorescence microscopy $(\sim 100-300 \%$ increases in nuclear localization using $25 \mu \mathrm{g} / \mathrm{ml} \mathrm{HA}$ ). $25 \mu \mathrm{g} / \mathrm{ml} \mathrm{HA}$ was used in (D). Note the colocalization of p-WWOX with p-Smad2/3 in the cytosol and their nuclear accumulation post HA treatment. Scale bar, $10 \mu \mathrm{m}$. E-G. Mouse breast cancer 4T1 cells (wild type and 2 cold-resistant variants), human monocytic THP-1 cells, and human leukemia Jurkat T cells were treated with HA $(25 \mu \mathrm{g} / \mathrm{ml})$ for indicated times. Nuclear accumulation of indicated proteins occurred in 10-20 min. $\mathrm{c}=$ medium control; $\alpha$-Tub $=\alpha$-Tubulin. 
Stimulation of p53-negative lung NCI-H1299 cells with highly purified HA (medical grade from Lifecore) for $1 \mathrm{hr}$ also resulted in nuclear accumulation of WWOX, p-WWOX (Tyr33 phosphorylation), p-ERK and Smads ( $\sim 100-300 \%$ increased), as determined by both Western blotting and immunofluorescence (Figures 1C, 1D). Colocalization of $\mathrm{p}$-WWOX with $\mathrm{p}-\mathrm{Smad} 2 / 3$ was shown in the cytoplasm, and HA stimulated translocation of these proteins to the nuclei (Figures 1C, 1D). Yet, a large portion of $\mathrm{p}-\mathrm{Smad} 2 / 3$ was present in the cytoplasm (Figures 1C, 1D).

We examined the responsiveness of WWOXexpressing cells to HA in time-course experiments. When murine breast cancer 4T1 cells, including 4T1-Luc, 4T1-Luc-c1d and 4T1-Luc-c5d, were exposed to HA for indicated times, ERK relocated to the nucleus in 10$20 \mathrm{~min}$ (Figure 1E). 4T1-Luc-c1d and 4T1-Luc-c5d are cold-resistant variants of 4T1-Luc. Human monocytic THP-1 and U937 cells, were treated with HA $(25 \mu \mathrm{g} / \mathrm{ml})$ for indicated times. Nuclear accumulation of ERK and pY216-Hyal-2 occurred in 10-20 min (Figure 1F; data not shown for U937). We generated antibody against Hyal-2 phosphorylation at Tyr216. The quality of this antibody is also shown (Figure 4A). Human leukemia Jurkat and
Molt-4 T cells were also treated with HA, which led to nuclear accumulation of ERK, p-ERK and pY287-WWOX in the nuclei in $20 \mathrm{~min}$ (Figure 1G; data not shown for Molt-4). Production of antibody against pY287-WWOX has been described [42]. Similar results were observed using T $\beta$ RII-deficient WWOX-expressing HCT116 cells [21] (data not shown).

\section{WWOX-negative cells are refractory to HA- induced nuclear relocation of Smad4 and other proteins}

By using WWOX-negative cells, we determined that these cells are refractory to HA-induced accumulation of Smads, p53, and other proteins of interest in the nucleus. Unlike MCF7, triple negative MDA-MB-231 and MDAMB-435S cells express little or no wild type WWOX, but MDA-MB-435S has WWOX2 expression [41]. Both MDA-MB-231 and MCF7 cells are responsive to TGF- $\beta 1$ mediated growth suppression [48]. Treatment of MDAMB-231 with HA did not effectively induce accumulation of p53 and Smad2/3 in the nucleus (Figure 2A; less than $10 \%$ for each indicated protein compared to the levels at time zero). Similarly, MDA-MB-435S cells were not
A

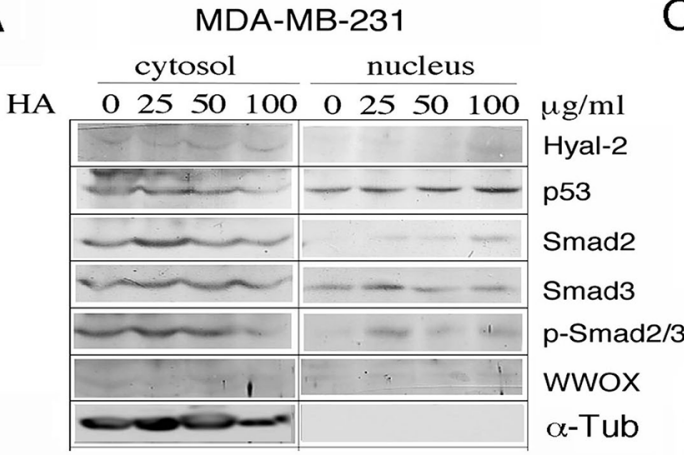

B

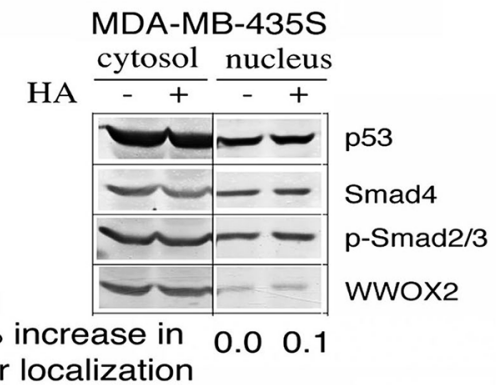

$\longrightarrow \alpha-T u b$

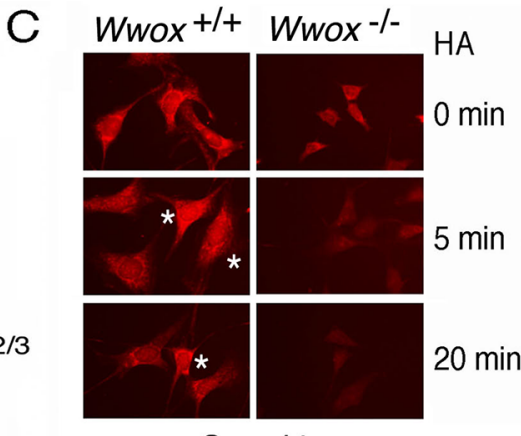

Smad4

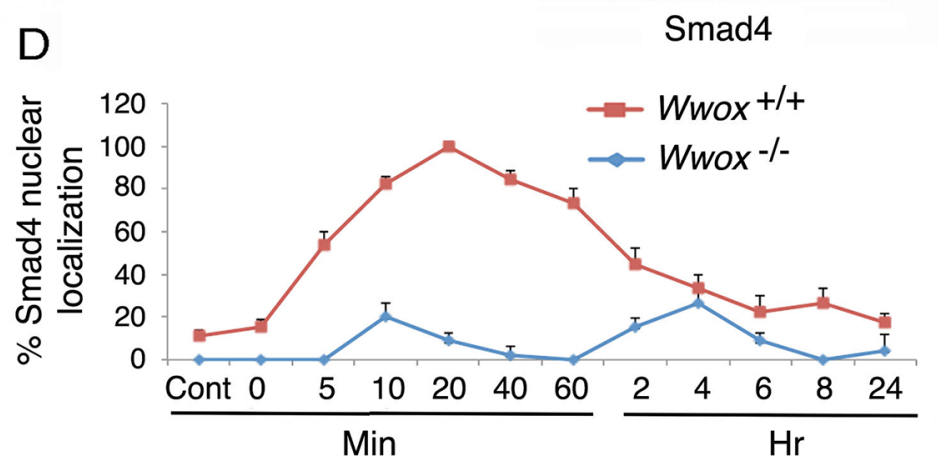

Figure 2: Wild type WWOX is necessary for HA induction of protein nuclear translocation. A, B. WWOX-deficient breast MDA-MB-231 and MDA-MB-435S were treated with high-molecular-weight HA (25 $\mu \mathrm{g} / \mathrm{ml})$ for $1 \mathrm{hr}$. Nuclear translocation of each indicated protein was retarded $\left(<10 \%\right.$ increase in nuclear localization compared to controls). C, D. Exposure of wild type $W w o x^{+/+}$ MEF cells to HA $(25 \mu \mathrm{g} / \mathrm{ml})$ resulted in relocation of endogenous Smad4 into the nucleus in 5 min (also see Supplementary Information Figures S3 and S5). When knockout $W$ wox $^{-/}$MEF cells were stimulated with HA, Smad4 appeared to relocate into nucleus in $4 \mathrm{hr}$ (also see Supplementary Figures S2-S4). The extent of Smad4 nuclear localization was quantified ( $\mathrm{n}=4 ; 20$ cells per count). Scale bar, $10 \mu \mathrm{m}$. 
responsive to HA-mediated nuclear translocation of WWOX2, p53, Smad4, and p-Smad2/3 (Figure 2B).

By immunofluorescence microscopy, HA induced relocation of a portion of endogenous Smad4 into the nuclei of wild type $W w x^{+++}$mouse embryonic fibroblast (MEF) cells in 5 min (see stars in Figure 2C), and the nuclear Smad4 appeared to migrate out to the cytoplasm 6 hr later (Figures 2C, 2D and Supplementary Information Figures S2 and S4). In stark contrast, when knockout Wwox ${ }^{-/}$MEF cells were stimulated with HA, endogenous Smad4 did not appear to relocate into nucleus (Figures 2C, 2D and Supplementary Information Figures S3 and S4). Presence of Smads in the nucleus of the aforementioned test cells is probably due to activation of the canonical TGF- $\beta$ pathway. A recent study showed that WWOX binds and sequesters Smad3 in the cytoplasm and thereby controls its transcriptional activity in the nucleus [49]. Loss of WWOX allows nuclear accumulation of Smads and other transcription factors to promote cancer growth [22, 27, 28, 49]. Overall, HA-induced protein nuclear accumulation is a universal phenomenon in cells, and is not related with the status of T $\beta$ RII, ER, AR and p53.

\section{Blebbistatin inhibits $\mathrm{HA}$-induced relocation of WWOX, ERK and JNK to the nucleus}

When native HA was pre-digested with hyaluronidase $\mathrm{PH}-20$, the degraded HA did not induce nuclear translocation of WWOX, p-WWOX, and p-ERK in L929 cells (Figure 3A). Similar results were observed in MCF7 and other WWOX-expressing cells (data not shown). Digestion of HA with Streptomyces hyalurolyticus hyaluronidase produced similar results. These observations suggest that integrity of HA is critical for the Hyal-2/WWOX/Smads signaling in cells. Alternatively, high molecular weight HA appears to exert mechanical stress to cell membrane, so that Hyal-2 may undergoes internalization to signal with WWOX and Smads. Cells were pre-exposed to blebbistatin, an inhibitor of myosin II, to relieve membrane stress, followed by treating with HA for $1 \mathrm{hr}$. Blebbistatin blocked HA-induced nuclear accumulation of ERK, JNK and pY33-WWOX in 4T1Luc and L929 cells (Figure 3B).

HA did not effectively cause relocation of cytosolic Smad3 to the nuclei (Figures 1A, 1D). In contrast to the aforementioned observations (Figure 3B), blebbistatin rapidly induced accumulation of Smad3 in the nucleus of DU145 cells and HA reversed the effect (Supplementary Figure S5). However, both HA and/or blebbistatin failed to induce accumulation of $\operatorname{Smad} 3$ in the nuclei in the wild type $W w^{\prime} x^{+/+}$MEF cells (data not shown).

\section{HA induces nuclear accumulation of Hyal-2}

We determined whether WWOX participates in HA-induced nuclear translocation of Hyal-2. By using generated polyclonal antibodies against Hyal-2 [21, 24], a 53-kDa protein is shown in breast cancer MCF7, neuroblastoma SK-N-SH, prostate cancer DU145, kidney fibroblast COS7 and L929 fibrosarcoma cells (Figure 4A). Exposure of these cells to HA reduced the expression of cytosolic Hyal-2 by approximately $50-90 \%$ (Figure 4A). We also produced antibody against Hyal2 phosphorylation at Tyr216. Androgen upregulated the expression of pY216-Hyal-2 in colon cancer HCT116 cells (Figure 4A). To further confirm the membrane localization of Hyal-2 [28, 31], immunostaining of nonpermeabilized COS7 cells showed the presence of cell surface Hyal-2 (Figure 4B). Nuclear staining with DAPI failed to reveal the nuclei (data not shown), indicating the integrity of these cells. Hyal-2 is clustered, in part, on the cell surface of COS7 and MDA-MB-231 cells (Figure 4B). In permeabilized HCT116, H1299, MCF7, DU145, COS7, L929 and other cells, Hyal-2 was also present on the cell membrane (data not shown) and in the cytoplasm $[9,21,24]$. We have shown that Hyal-2 translocates from the lysosomes to the mitochondria during staurosporinemediated apoptosis, suggesting that Hyal-2 participates in damaging to mitochondria during apoptosis [24].

By stimulating wild type $W$ ox $^{+/+}$MEF cells with HA, endogenous Hyal-2 rapidly translocated to the nucleus in 5 min (Figure 4C). Interestingly, endogenous Hyal-2 mainly localized in the nucleus of knockout $W w^{-1 /} \mathrm{MEF}$ cells $(\sim 60 \%)$. Reduction of nuclear accumulation of Hyal2 occurred in 20-40 min post HA stimulation (Figure 4C). The observations suggest that WWOX limits relocation of Hyal-2 to the nucleus.

\section{HA increases the complex formation of Hyal-2, WWOX and Smads, followed by reduction}

We examined the complex formation between Hyal-2 and WWOX. By immunoprecipitation using WWOX antibody, HA increased the binding of WWOX with Hyal-2 in the cytosol in $30 \mathrm{~min}$ in THP-1 cells, whereas at this point the complex remained in the cytosol and no apparent translocation to the nucleus was shown (Figure 4D). In contrast, HA induced nuclear translocation of the WWOX/Hyal-2 complex in U937 cells, and the level of the complex in the cytoplasm was reduced (Figure 4D). In resting SK-N-SH cells, there was a cytosolic Hyal-2/WWOX/Smads complex, whereas HA reduced the level of the complex, as determined by co-immunoprecipitation using Hyal-2 antibody (Figure $4 \mathrm{E})$. The reduction is due to relocation of Hyal-2 to the nucleus. In parallel, immunoprecipitation by Smad3 antibody revealed the presence of the Hyal-2/WWOX/ Smads complex. However, due to nuclear relocation, coimmunoprecipitation by Smad3 antibody failed to show the Hyal-2/WWOX/Smads complex (Figure 4E).

Similarly, WWOX bound Hyal-2 and ERK in resting Jurkat T and TNF-resistant L929R cells, and HA reduced 
the WWOX/Hyal-2/ERK complex formation in the cytosol in $30 \mathrm{~min}$, suggesting nuclear translocation of the complex (Figure 4F). Non-immune sera did not precipitate the aforementioned complexes (data not shown). We have recently demonstrated the binding interaction between WWOX with ERK and $\mathrm{I} \kappa \mathrm{B} \alpha$ [42]. Similar results were observed with THP-1 and U937 monocytic cells and TNFsensitive L929 cells (data not shown).

A plasmid construct expressing an EGFP-tagged dominant negative WWOX with alterations at the $N$ terminal WW domain (dn-WW) was made [37, 39].
The dominant negative suppresses stress stimuliinduced Tyr33-phosphorylation in WWOX and Ser46phosphoryltaion in p53, and p53/WWOX-mediated apoptosis [37, 39]. By immunoprecipitation using antiHyal-2 antibody, we demonstrated the binding of Hyal-2 with WWOX, Smad4 and p-Smad2/3 in the control EGFPexpressing COS7 cells (Figure 4G). Ectopic dn-WW reduced the binding of Smads and WWOX with Hyal2 (Figure 4G), suggesting that dn-WW reduces Tyr33 phosphorylation in WWOX to cause dissociation. Again, HA dissociated the Hyal-2/WWOX/Smad4 complex
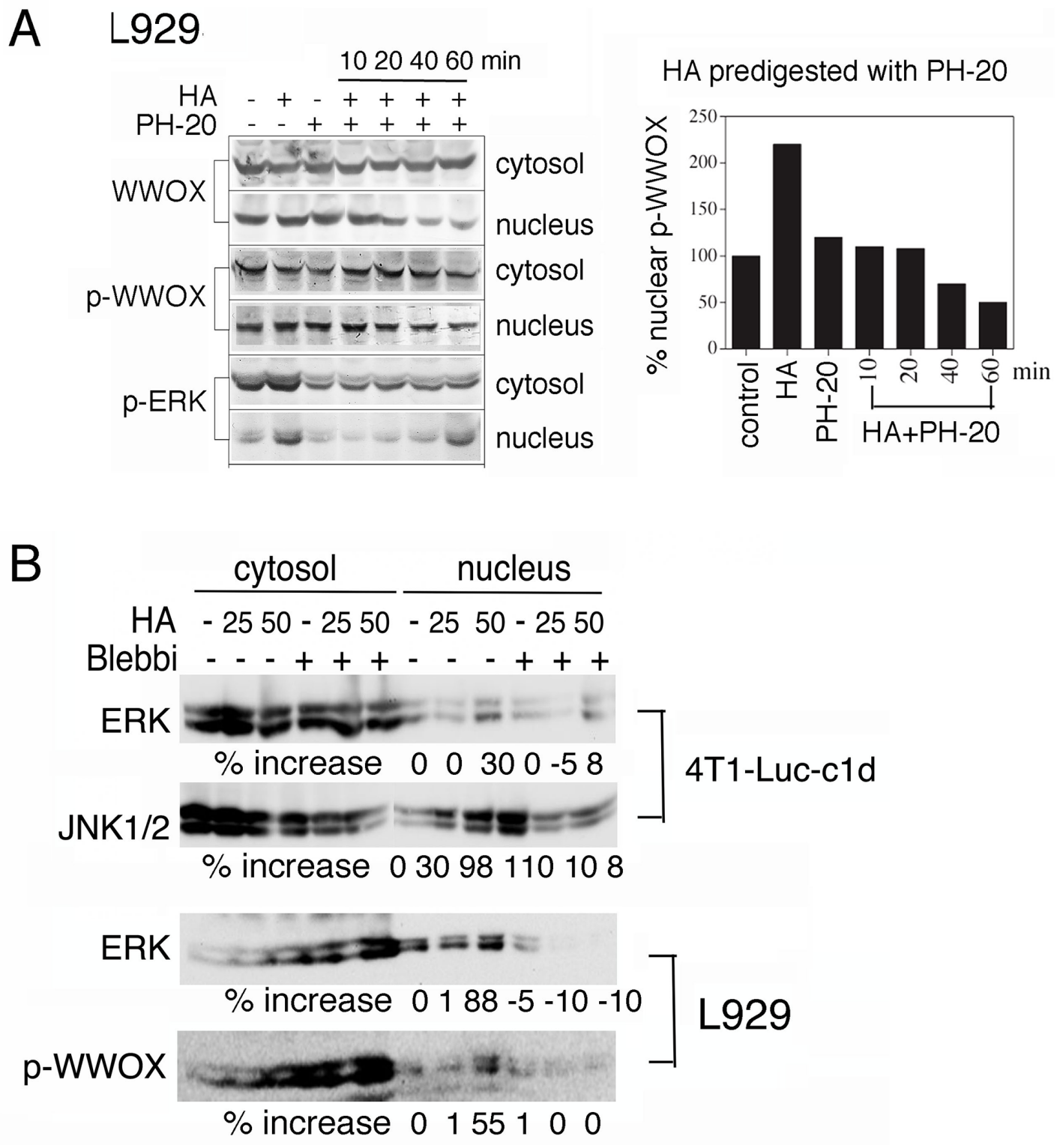

Figure 3: Blebbistatin blocks HA-mediated protein nuclear translocation. A. HA (100 $\mu \mathrm{g} / \mathrm{ml})$ was predigested with PH-20 (100 units $/ \mathrm{ml}$ ) for various indicated times. The digested HA could not induce nuclear translocation of WWOX, p-WWOX and p-ERK in L929 cells (representative data from 2 experiments). The extent of p-WWOX nuclear translocation is quantified (right panel). B. L929 and 4T1-Luc-c1d cells were pre-treated with blebbistatin $(10 \mu \mathrm{M})$ for $1 \mathrm{hr}$, followed by exposure to HA $(25 \mathrm{and} 50 \mu \mathrm{g} / \mathrm{ml})$ for determining the relocation of indicated proteins to the nucleus by Western blotting. Blebbistatin blocked HA-induced nuclear relocation of ERK, JNK and pY33-WWOX. p-WWOX = pY33-WWOX; Blebbi = blebbistatin. 
A

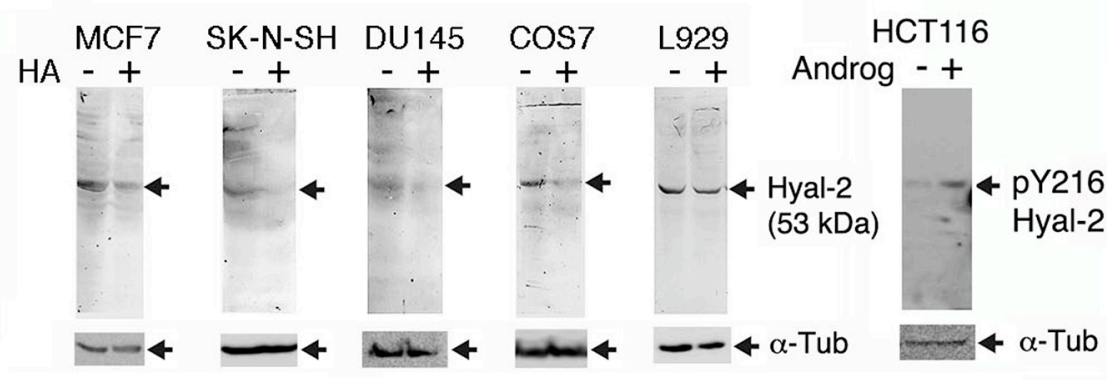

$\mathrm{B}$

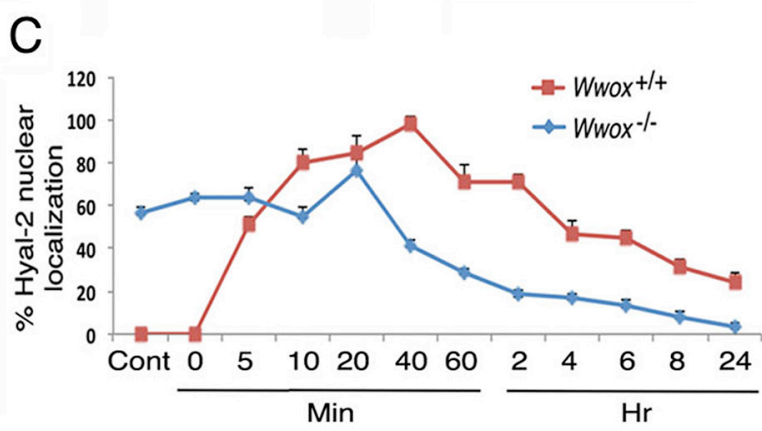

D

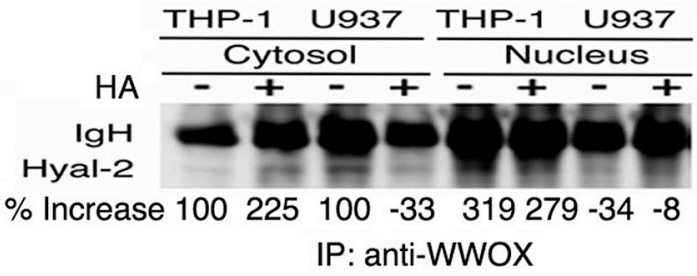

E

F

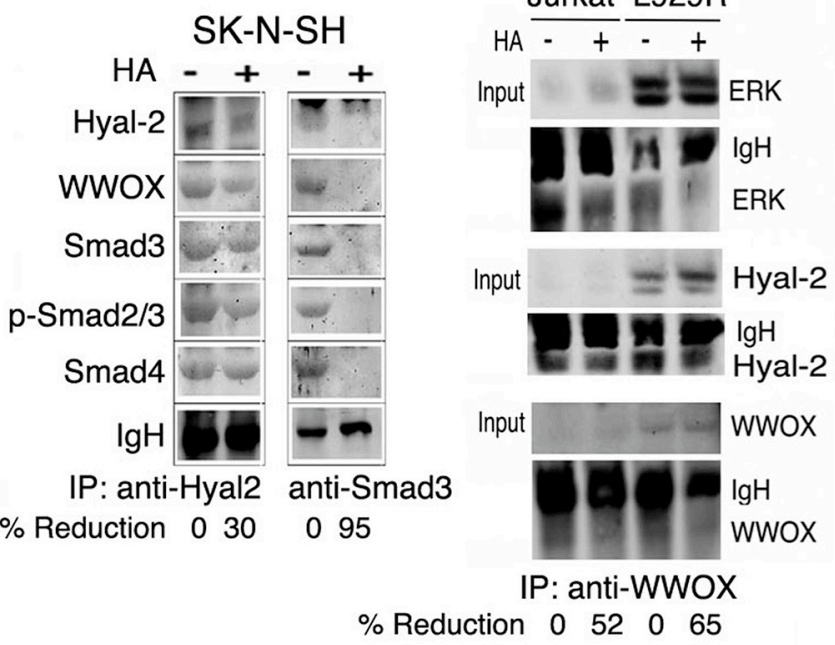

G

$\cos 7$

$\mathrm{H}$

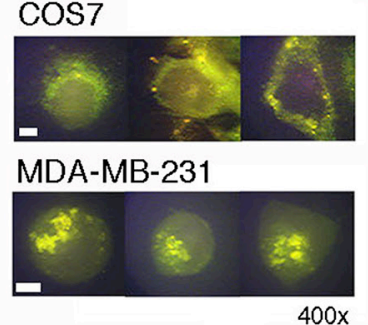

HA $\frac{\text { egfp }}{-+} \frac{\text { dn-WW }}{-+}$

egfp dn-WW

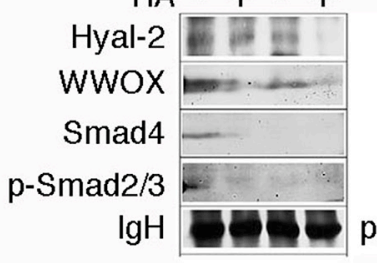

IP: anti-Hyal-2

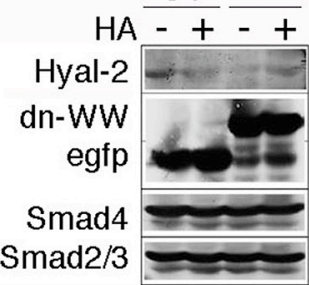

Input

\% Reduction 0857895

Figure 4: HA increases the complex formation of Hyal-2, WWOX and Smads, followed by reduction. A. Indicated cell lines were treated with HA $(50 \mu \mathrm{g} / \mathrm{ml})$ for $30 \mathrm{~min}$ at $37^{\circ} \mathrm{C}$. Cytosolic fractions were used for reducing SDS-PAGE and Western blotting. HCT116 cells were treated with androgen $(100 \mathrm{ng} / \mathrm{ml})$ for $30 \mathrm{~min}$, which led to increased pY216-Hyal-2 expression. B. Immunostaining of non-permeabilized COS7 cells shows the expression of Hyal-2 on cell surface. Hyal-2 is clustered on the surface of non-permeabilized MDAMB-231 cells. Scale bar, $5 \mu \mathrm{m}$. C. HA $(25 \mu \mathrm{g} / \mathrm{ml})$ rapidly induced translocation of Hyal-2 to the nucleus in the wild type $W w o x^{+/+}$MEF cells, followed by reduction. In the knockout $W w o x^{-/}$MEF cells, approximately $60 \%$ of endogenous Hyal- 2 is present in the nucleus. HA reduces the nuclear localization. D. By immunoprecipitation using WWOX antibody, HA increased the WWOX/Hyal-2 complex in the cytosol, whereas the nuclear level of the complex was still low in THP-1 cells. In U937 cells, HA reduced the cytosolic WWOX/Hyal-2 complex in 30 min and showed the increased complex in the nucleus. E. HA $(25 \mu \mathrm{g} / \mathrm{ml})$ reduced the cytosolic Hyal-2/WWOX/Smads complex in SK-N-SH cells in $30 \mathrm{~min}$, as determined by immunoprecipitation using Hyal-2 antibody. Immunoprecipitation by Smad3 antibody revealed the presence of the Hyal-2/WWOX/Smads complex in resting cells and HA decreased the complex. F. Jurkat T cells and L929R fibroblasts were treated with HA $(25 \mu \mathrm{g} / \mathrm{ml})$ for $30 \mathrm{~min}$, followed by processing immunoprecipitation with WWOX antibody. HA reduced the complex formation of cytosolic WWOX/Hyal-2/ERK. G. HA reduced the complex formation of Hyal-2/WWOX/Smad4 in EGFP-expressing COS7 cells. Also, ectopic EGFP-dn-WW suppressed the complex formation. In the input, one-tenth amounts of the cell lysates were loaded in the SDS-PAGE. H. The Hyal-2/WWOX/Smad4 complex was not affected by hyaluronidase PH-20 treatment of MCF7 cells for $1 \mathrm{hr}$. 
(Figure 4G). In MCF7 cells, hyaluronidase PH-20 alone did not abolish the Hyal-2/WWOX/Smads complex, and that hyaluronidase-degraded HA had a reduced effect on the complex (Figure 4H).

\section{Yeast two-hybrid analysis for determining domain/domain binding for the WWOX/Hyal-2/ Smad4 complex}

To further substantiate the above observations, we mapped the domains in the WWOX that interacts with Smad4 and Hyal-2. By utilizing Ras rescue-based yeast twohybrid analysis [16, 21, 37, 39, 42], Smad4 was anchored onto the cell membrane as target, and various WWOX constructs were expressed in the cytoplasm as baits. WWOX physically bound Smad4 via its $N$-terminal first WW domain (Figure 5A), as evidenced by the growth of yeast at $37^{\circ} \mathrm{C}$ using a selective agarose plate containing galactose. No binding interaction was observed using a phosphorylation mutant of WW domain, WWOXww(Y33R), suggesting an essential role of $\mathrm{Y} 33$ phosphorylation in the binding.

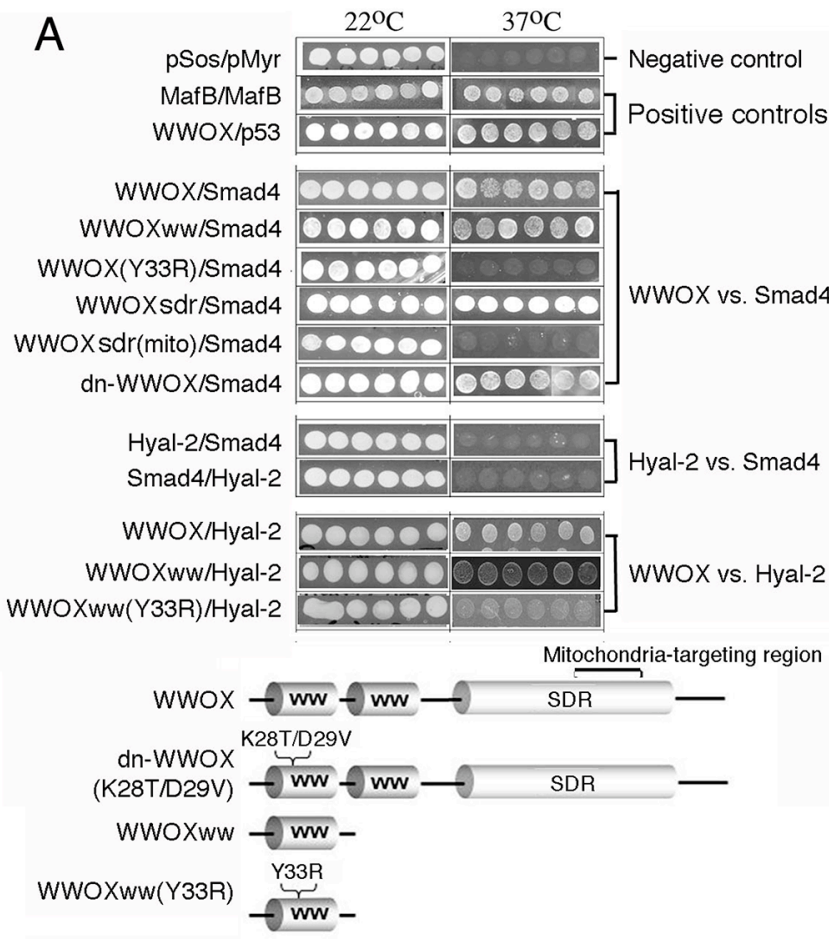

The $C$-terminal SDR domain of WWOX also bound Smad4, whereas the mitochondria-targeting region in SDR domain did not bind Smad4 (Figure 5A). dn-WWOX, with alterations at K28 and D29 (K28T/D29V) [37], was capable of binding Smad4 (Figure 4), suggesting that Smad4 binds both the SDR domain and the pY33-WW domain. Compared to wild type murine Smad4 (60 kDa), our truncated Smad4 (43 kDa) has a $C$-terminal deletion from amino acid \#392 to 551 (Genbank accession AY493561) [21].

In agreement with our previous report [21], the first WW domain of WWOX bound Hyal-2 (Figure $5 \mathrm{~A})$. Alteration of the conserved Tyr33 phosphorylation site abolished the binding (Figure 5A). In contrast, Hyal-2 could not bind Smad4 (Figure 5A), indicating that WWOX connects the binding of Hyal-2 with Smad4. Results from co-immunoprecipitation support these observations (Figures 4D-4G). As a negative control, no binding interactions were observed for the empty $\mathrm{pSos} / \mathrm{pMyr}$ vectors. In positive controls, WWOX/p53 binding and MafB self-interaction were observed (Figure 5A).

B
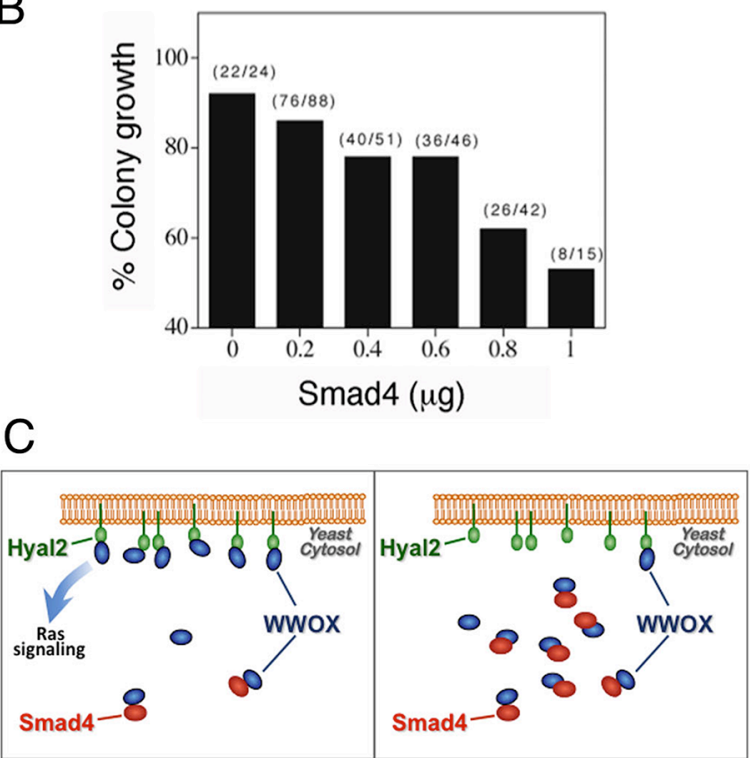

Figure 5: Tyr33 phosphorylation of WWOX is essential for interacting with Smad4 and Hyal-2, and Smad4 competitively blocks the binding of WWOX to membrane Hyal-2. A. Ras rescue-based protein/protein interaction in the cytoplasm by yeast two-hybrid was performed [16, 21, 37, 39]. In positive controls, binding of WWOX with p53 and MafB self-interaction are shown, as evidenced by the growth of yeast at $37^{\circ} \mathrm{C}$ using a selective agarose plate containing galactose. No yeast growth at $37^{\circ} \mathrm{C}$ was observed for the empty pSos/pMyr vectors in negative controls. The $N$-terminal first WW domain of WWOX (WWOXww) bound Smad4 and Hyal-2. Alteration of Tyr33 to Arg33 in the first WW domain, WWOXww(Y33R), abolished its interaction with Smad4 and Hyal-2. Dominant negative WWOX, dn-WWOX(K28T/D29V), also bound Smad4. The mitochondria-targeting area (amino acid 209-273) of the $C$-terminal SDR domain did not bind Smad4. No direct interaction was observed for Hyal-2 and Smad4. B, C. In this competitive binding assay, yeast cells were transfected with Hyal-2 (target; green) for anchoring onto cell membrane, in the presence of a constant amount of WWOX (bait; dark blue) and various amounts of $\operatorname{Smad} 4$ (competitor; red). $\%$ Colony growth $=\left(\right.$ survival colonies at $\left.37^{\circ} \mathrm{C}\right) /\left(\right.$ total colonies grown at $22^{\circ} \mathrm{C}$ ) (see brackets). Smad4 competitively blocked the binding of WWOX to membrane Hyal-2, and thereby prevented yeast growth. 


\section{Hyal-2 competes with Smad4 in binding to WWOX}

The aforementioned results revealed that both Hyal-2 and Smad4 bound to the $N$-terminal pY33-WW domain in WWOX, although Samd4 can also bind the C-terminal SDR domain (Figure 5A). To test whether there is a competitive binding between Hyal-2 and Smad4 to WWOX, yeast cells were transfected with membrane-anchored Hyal-2 (target), in the presence of a fixed amount of WWOX (bait) and various amounts of Smad4 (competitor) in the cytoplasm. Smad4 competitively blocked the binding of Hyal-2 with WWOX (Figures 5B, 5C). That is, once the p-WWOX/ Smad4 complex is formed in the cytoplasm, the complex may have a reduced binding for membrane Hyal-2 and thereby prevents yeast cell growth at $37^{\circ} \mathrm{C}$ (Figures $5 \mathrm{~B}, 5 \mathrm{C})$.

\section{dn-WWOX blocks HA-induced nuclear accumulation of Smads}

To further verify the observations that WWOX connects the binding interaction between Hyal-2 and Smads, dn-WWOX was utilized to block the WWOX function. COS7 cells were transfected with the expression construct of EGFP-dn-WWOX or EGFP by electroporation, cultured $48 \mathrm{hr}$, and then treated with HA for $30 \mathrm{~min}$. dnWWOX blocked the HA-induced nuclear translocation of p-Smad2/3 and Smad4 (Supplementary Figure S6).

\section{Agonist anti-Hyal-2 antibody and antisense Hyal-2 mRNA stimulate nuclear translocation of WWOX and Smads}

Whether Hyal-2 antibody could induce activation of WWOX and Smads was examined. Exposure of NCI-H1299 cells to diluted anti-Hyal-2 antiserum for $30 \mathrm{~min}$ resulted in spontaneous nuclear translocation of WWOX and Smads (Figure 6A). In contrast, nonimmune serum and culture medium had no effects (Figure 6A). These results were further confirmed with L929 and HCT116 cells (data not shown). Furthermore, suppression of Hyal-2 expression by antisense mRNA spontaneously induced nuclear translocation of WWOX and Smads in T $\beta$ RII-deficient HCT116 cells (Figure 6B). Without Hyal-2, HA could not further induce nuclear translocation of WWOX and Smads (Figure 6B). These results were also observed when other types of cells were tested (data not shown). Taken together, HA increases the complex formation of Hyal-2 with pY33WWOX and Smads (Figure 6C). The Hyal-2/WWOX/ Smads complex relocates to the nucleus. While a portion of WWOX and Hyal-2 are in the lysosome, formation of the Hyal-2/WWOX/Smads complex is expected to occur in response to $\mathrm{HA}$.
Time-lapse FRET microscopy of HA-induced signaling involving Smad4/WWOX/p53 for membrane blebbing and Smad4/Hyal-2/WWOX for bubbling cell death

We examined HA-induced signaling by timelapse tri-molecular FRET microscopy [42]. DU145 cells were transfected with ECFP-Smad4, EGFP-WWOX and DsRed-monomer-p53 expression constructs. The energy emitted from ECFP goes to EGFP and finally to DsRed monomer. That is, ECFP-Smad4 was excited to allow the energy transfer down to EGFP-WWOX and then to DsRedmonomer-p53. WWOX acts as a bridge for Smad4 and p53 in the energy transfer or signaling. Positive signals were observed in HA-stimulated DU145 cells for the Smad4/ WWOX/p53 signaling (Figure 7A and Supplementary Video 1). By merging the resulting FRET signals with the bright field imaging, we found that cells exhibited membrane blebbing without cell death (see star in the left panel, Figure $7 \mathrm{H}$ and Supplementary Video 2). Both dn-WWOX and dn-p53 abolished the signaling event (Figures 7B, 7C and Supplementary Videos 3 and 4). We have previously reported that membrane blebbing is an occurrence for cells to resist death, rather than committing them to die [50].

In another signaling, HA activated the signaling of ECFP-Smad4, EGFP-Hyal-2(-sp) and DsRed-monomerWWOX (Figure 7D and Supplementary Video 5). EGFP-Hyal-2(-sp) is devoid of the GPI linkage and is for intracellular expression. Notably, HA-treated cells underwent bubbling cell death (see star in the right panel, Figure $7 \mathrm{H}$ and Supplementary Video 6). We have recently reported UV/cold shock-induced non-apoptotic bubbling cell death - one bubble per cell independently of caspase activation [51, 52]. Antisense mRNA-targeting Hyal-2 abolished the signaling (Figure 7E and Supplementary Video 7). Also, dn-WWOX abolished the singling event (Figure 7F and Supplementary Video 8). In negative controls, HA failed to induce signaling in cells expressing ECFP, EGFP and DsRed (Figure 7G and Supplementary Video 9). We have recently demonstrated that calcium ionophore A23187 and phorbol myristate ester activates the signaling of I $\kappa \mathrm{B} \alpha / \mathrm{ERK} / \mathrm{WWOX}$ in lymphocytic cells

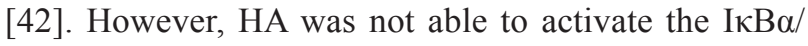
ERK/WWOX signaling (Figure $7 \mathrm{H}$ and Supplementary Video 10).

\section{Immunoelectron microscopy of HA-induced nuclear relocation of WWOX and Smad4}

When HCT116 cells were stimulated with HA for 60 min, both WWOX and Samd4 were shown to localize in the nucleus, as determined by immunoelectron microscopy [21] (Figure 8). However, little or no Hyal-2 was found (Figure 8). Indeed, without using Hyal-2 antibody, HA had a much less activity in inducing nuclear accumulation 
of WWOX, Hyal-2 and Smad4 in HCT116 cells, as determined by Western blotting (data not shown). TGF- $\beta 1$ induced nuclear localization of WWOX and Smad4 in HCT116 cells, and the nuclear level of Hyal-2 was low also (Figure 8). Pre-stimulation of cells with HA for $20 \mathrm{~min}$, followed by stimulating with TGF- $\beta 1$ for $1 \mathrm{hr}$, resulted in a dramatic increase in the complex formation of WWOX, Hyal-2 and Smad4 in the nucleus (Figure 8). In agreement with our previous observations [21], TGF- $\beta 1$ participates in the activation of the WWOX/Hyal-2/Smad4 complex.
Hyal-2, WWOX and Smad4 induce apoptosis synergistically, and suppression of WWOX by siRNA abrogates Smad4-mediated apoptosis

Both WWOX and Smad4 are tumor suppressors and proapoptotic proteins [17, 21, 27-31, 37]. We determined whether ectopic Hyal-2, Smad4, and WWOX induce cell death synergistically. L929 cells were electroporated with Hyal-2, WWOX, and/or Smad4 DNA constructs and cultured $48 \mathrm{hr}$, followed by cell cycle analysis using flow cytometry. As determined
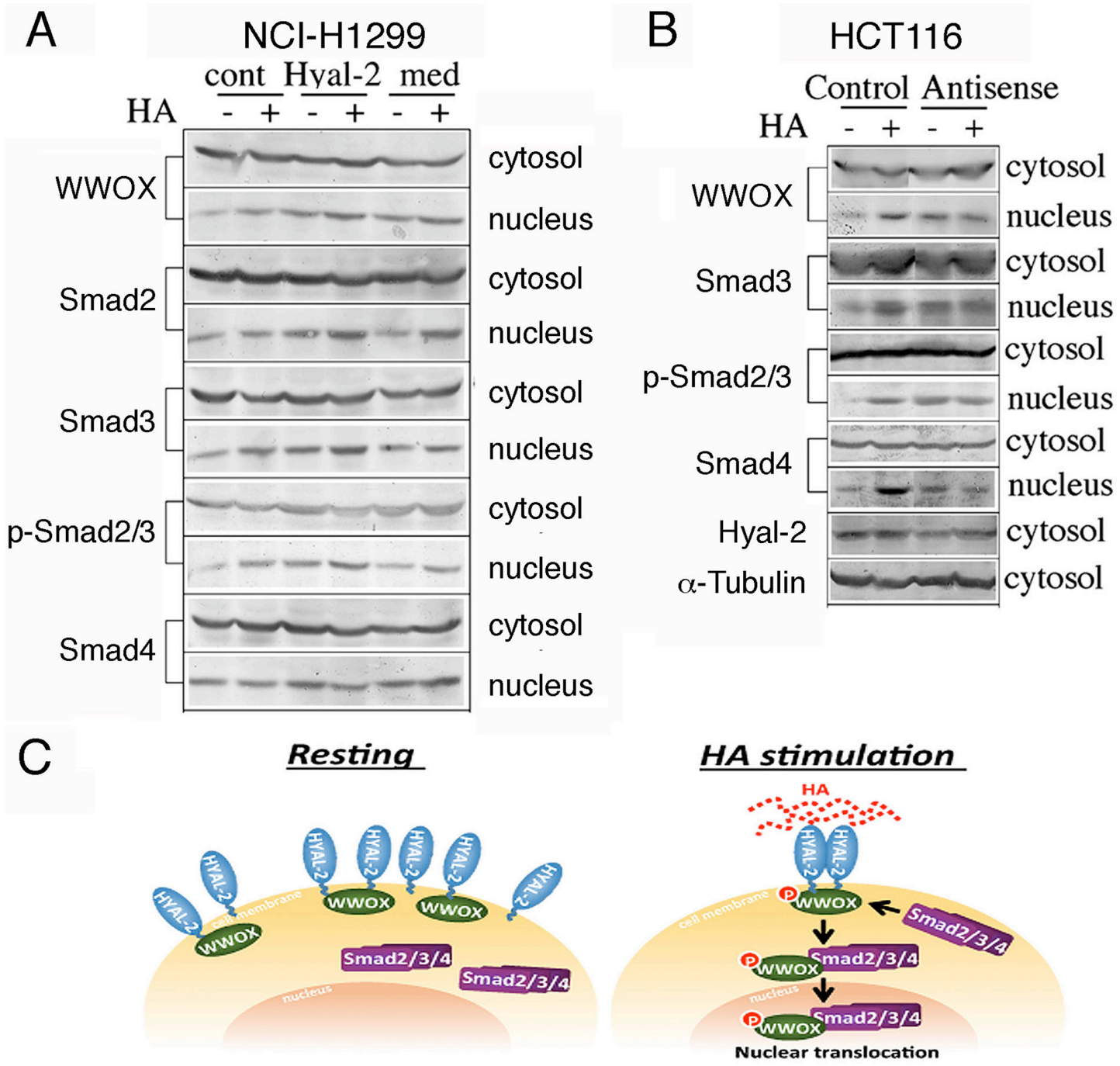

\section{HA stimulation}

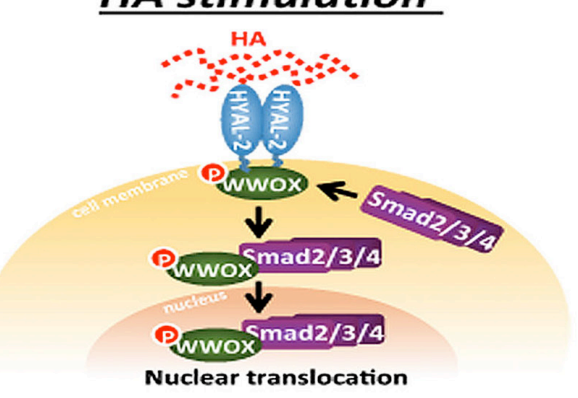

Figure 6: Induction of WWOX and Smads nuclear accumulation by agonist Hyal-2 antibodies and antisense mRNA. A. Compared to non-immune serum (cont) and medium control (med), exposure of NCI-H1299 cells to agonist antibodies against Hyal-2 (1:1000 dilution) for 30 min resulted in spontaneous nuclear translocation of WWOX and Smads ( 75-200\% increase in nuclear localization; see the nuclear protein levels without HA treatment; representative data from 2 experiments). These cells were further exposed to HA (100 $\mu \mathrm{g} / \mathrm{ml})$ for $30 \mathrm{~min}$. B. Similarly, suppression of Hyal-2 expression by antisense mRNA ( $70 \%$ reduction) spontaneously induced nuclear translocation of WWOX and Smads in T $\beta$ RII-deficient colon HCT116 cells. Without Hyal-2, HA could not increase nuclear translocation of these proteins ( $\sim 100-200 \%$ increase in nuclear localization; see the nuclear protein levels without HA treatment; representative data from 2 experiments). C. In resting cells, Hyal-2 binds WWOX in the cell membrane or in the lysosome. HA increases the formation of the Hyal-2/ WWOX/Smads complex for relocating to the nucleus. Without WWOX, Hyal-2 may spontaneously accumulate in the nucleus. 
above, Hyal-2 could not directly bind Smad4 (Figure 5). As expected, Hyal-2 could not effectively enhance the apoptotic function of Smad4 (Figure 9A). The extent of apoptosis and growth suppression was measured at the subG1 and G1 phases of the cell cycle, respectively. In agreement with our previous observations [15], Hyal-2 enhanced WWOX-mediated apoptosis, up from $30 \%$ to $60 \%$ (Figure $9 \mathrm{~B}$ ). When in combination, Hyal-2, WWOX and Smad4 greatly increased the cell death (Figure 9B). The inverse relationship in the G1 phase, as opposed to SubG1 phase, was also observed (Figure 9B).

We have made plasmid constructs expressing small-interfering RNA (siRNA)-targeting WWOX (WWOXsi in pSuppressorNeo) [41]. Ectopic WWOXsi blocked Smad4-mediated growth inhibition and apoptosis (Figure 9C). In controls, scramble siRNA had no effect (Figure 9C). Opposite effect was also seen in the G1 phase. These observations suggest that WWOX acts synergistically with Smad4 in causing apoptosis. In parallel, ectopic WWOX and Hyal-2 enhance the transcription activation of Smad4 for SMAD-regulated promoter, thereby inducing apoptosis [21].

\section{Traumatic brain injury involves in nuclear accumulation of Hyal-2 and WWOX}

Finally, we determined the potential role of WWOX and Hyal-2 in apoptosis in vivo. In a traumatic brain injury, rats were pierced with needles into their brains [40, 53]. We determined that post injury for 3 and $24 \mathrm{hr}$, there were increased numbers of apoptotic neurons in the brain cortex. And, the complex formation of WWOX/Hyal-2
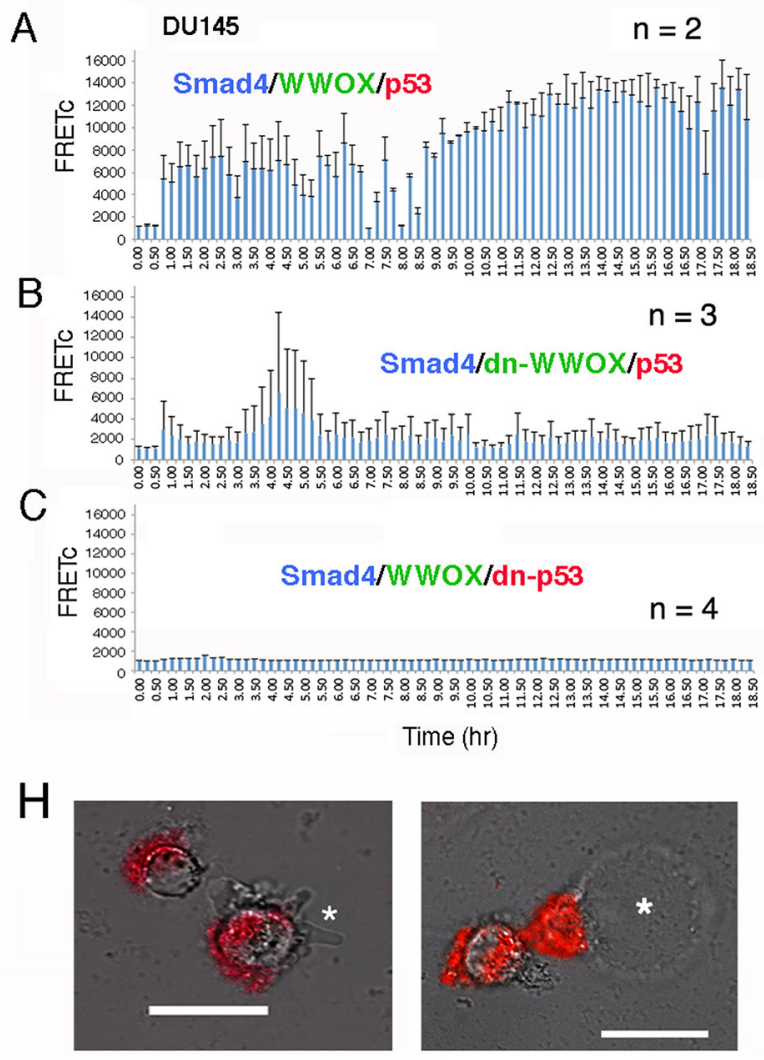

Smad 4/WWOX/p53

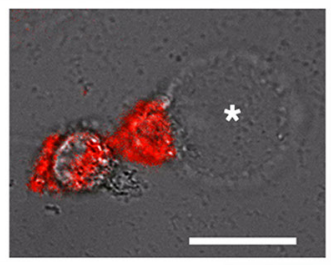

Smad4/Hyal-2(-sp)/WWOX

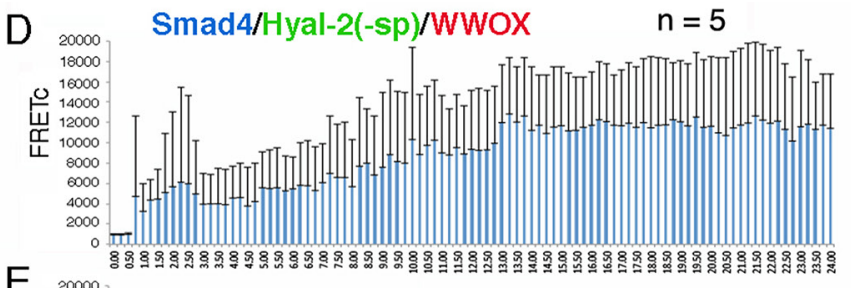

E

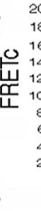

\begin{tabular}{c}
20000 \\
18000 \\
1000 \\
1400 \\
1200 \\
1000 \\
800 \\
800 \\
400 \\
200 \\
\\
2000 \\
\hline
\end{tabular} ?

\section{$\mathrm{F}$}

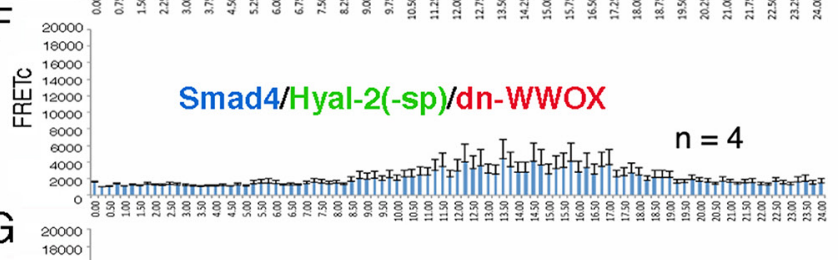

$G$

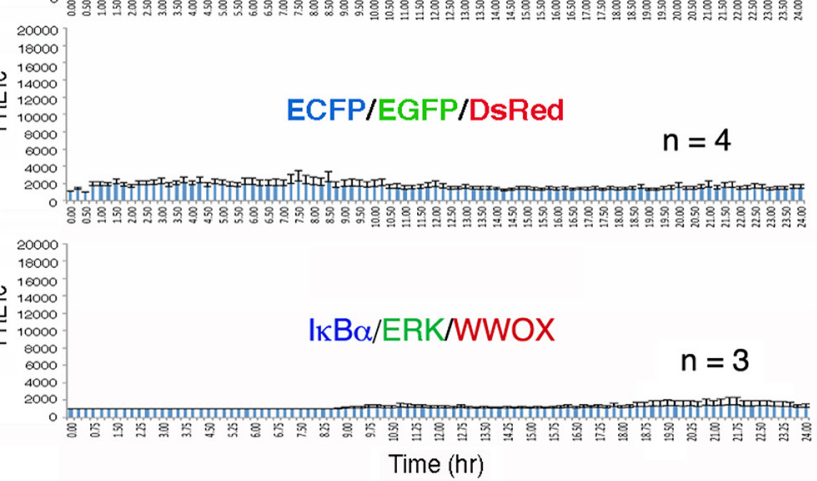

Figure 7: Time-lapse FRET microscopy for HA-activated signaling pathways. A. DU145 cells were transiently transfected with ECFP-Smd4, EGFP-WWOX and DsRed-monomer-p53 expression constructs. HA $(25 \mu \mathrm{g} / \mathrm{m})$ induced energy transfer from ECFPSmad4 to EGFP-WWOX and then to DsRed-monomer-p53 for the Smad4/WWOX/p53 signaling. Data are shown as FRETc (FRET concentration). B, C. Dominant negatives for WWOX and p53 abolished the signaling. D. HA also induced signaling for ECFP-Smd4, EGFP-Hyal-2(-sp) and DsRed-monomer-WWOX. Cytosolic EGFP-Hyal-2(-sp) is devoid of the GPI linkage. E, F. Both antisense mRNA targeting Hyal-2 and dn-WWOX abolished the HA-induced signaling. G. In negative controls, HA did not induce signaling in cells expressing ECFP, EGFP and DsRed. H. The Smad4/WWOX/p53-expressing cells started to undergo membrane blebbing post stimulation with HA for $1.5 \mathrm{hr}$ (see the star in the left). No cell death occurred. In contrast, Smad4/Hyal-2/WWOX-expressing cells underwent bubbling cell death [51, 52], post HA stimulation for 5-16 hr (see the star for the generated bubble in the right). Also, see Supplementary Video

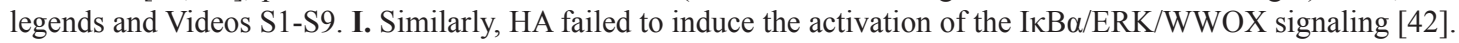


was increased with time and accumulated in the apoptotic nuclei of neurons (Figure 10).

\section{DISCUSSION}

We have previously determined that TGF- $\beta 1$ induces the signaling of the Hyal-2/WWOX/Smad4 complex for transcriptional activation of SMADresponsive element [21]. When the SMAD-responsive element is overly activated, cell death occurs [21]. In this study, we determined by yeast two-hybrid analysis that WWOX acts as a bridge to bind and connect both Hyal-2 and Smad4. Hyal-2 binds to the $N$-terminal Tyr33-phosphorylated first WW domain, and Smad4 binds to the SDR domain. Since Smad4 also interacts with the first WW domain, there is a competitive binding interaction between Smad4 and Hyal-2 to WWOX.
High molecular weight HA increases the formation of endogenous Hyal-2/WWOX/Smad4 complex rapidly, followed by relocating to the nuclei in 20-40 $\mathrm{min}$, in WWOX-expressing normal and cancer cells. No apparent cell death occurs. In WWOX-deficient cells, HA cannot effectively induce nuclear translocation of Smads, whereas Smad4 may spontaneously migrate to the nucleus. Stimulation of membrane Hyal-2 by specific antibody results in accumulation of WWOX and Smads in the nucleus. Furthermore, knockdown expression of Hyal-2 also increases nuclear accumulation of WWOX and Smads. These observations suggest that WWOX and Hyal-2 coordinate synergistically in relocating to the nucleus. Alternatively, WWOX and Hyal-2 may mutually sequester each other for nuclear relocation. WWOX is known to sequester Smad3 in the cytoplasm and inhibits its transcriptional function [48]. In a recent study, we determined that trafficking protein TRAPPC6A is a
A

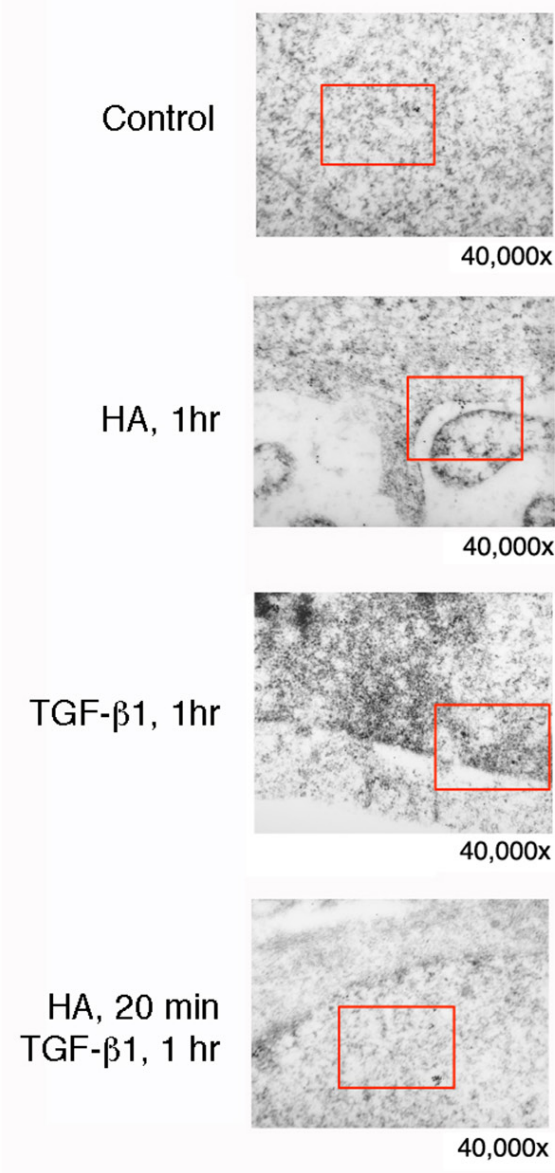

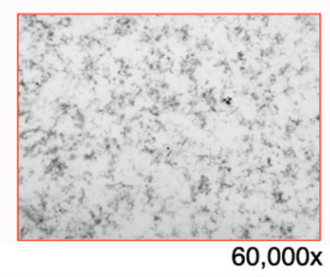
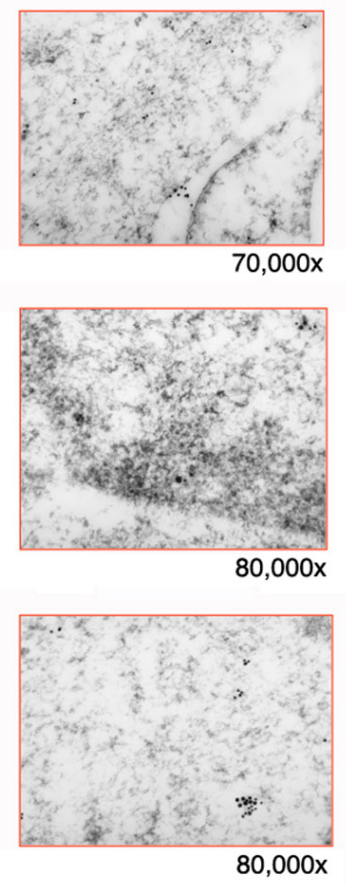

Hyal-2: $10 \mathrm{~nm}$

WWOX: $15 \mathrm{~nm}$
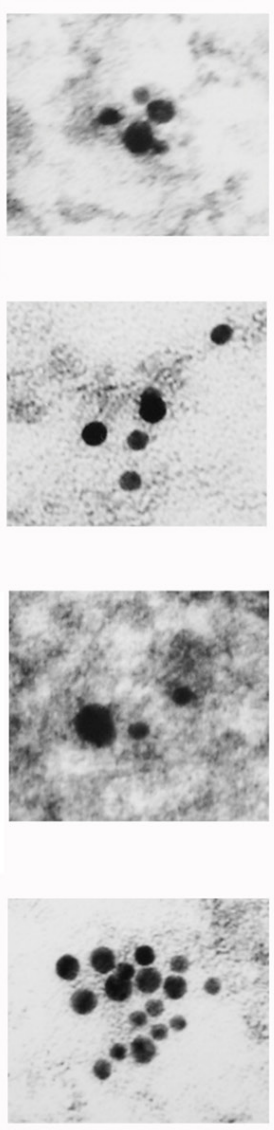

Nucleus
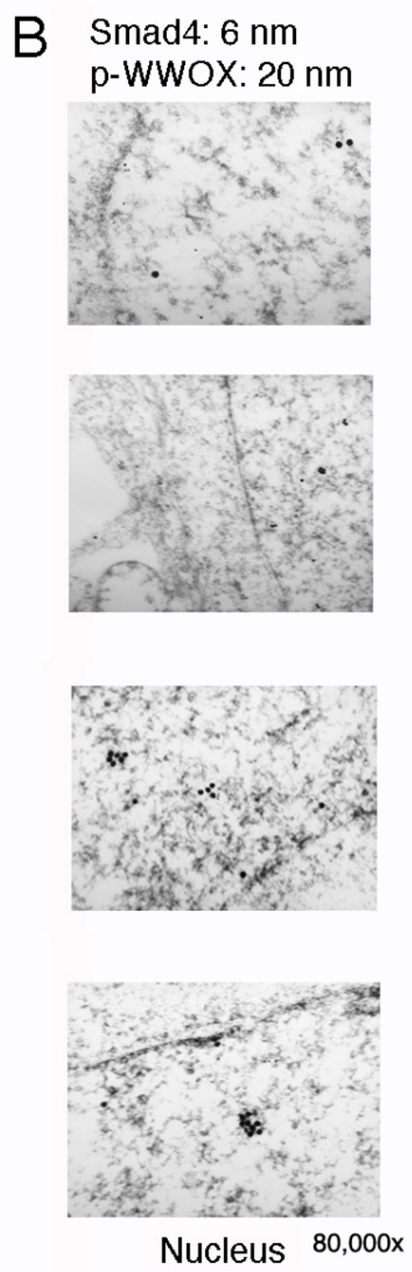

Figure 8: Immunoelectron microscopy analysis for HA induction of WWOX/Hyal-2 nuclear translocation. A. HCT116 cells were treated with HA for 20 min and then subjected to processing for immunoelectron microscopy using antibodies against WWOX and Hyal-2, plus indicated immunogold particles. Where indicated, the enlarged areas were from 40,000 to 80,000x magnifications. Shown in the column at right are digitally enlarged immunogold particles in the nuclei. B. Immunostaining for p-WWOX and Smad4 particles is shown. 
carrier for WWOX to undergo nuclear translocation [5456]. Whether the TRAPPC6A/WWOX complex acts as a vehicle for Hyal-2 relocation to the nucleus remains to be established. In addition, whether WWOX interacts with AMOT (Angiomotin), a tight junction protein in the cell membrane/cytoskeletal area [27], during signaling remains to be determined.

To prove the HA-induced signaling event, we have carried out real time tri-molecular FRET analysis [42]. HA induces the signaling pathways for ectopic Smad4/ WWOX/p53 and Smad4/Hyal-2/WWOX complexes. When the binding of Smad4, Hyal-2 and WWOX increases with time and the complex localizes in the nucleus, cells undergo bubbling cell death [51, 52]. Bubbling cell death is not apoptosis and is defined as "formation of a single nitric oxide-containing bubble from the nucleus per cell and release of this swelling bubble from the cell surface to extracellular space that causes cell death." Bubbling cell death is caspaseindependent, and exhibits no DNA fragmentation and flip-over of phosphatidylserine. In contrast, HA increases the binding affinity of Smad4/WWOX/p53 with time, and causes membrane blebbing but without cell death. We have designed the signaling in a reverse way. That is, the signaling starts from Smad4. In this manner, we know the complex has already formed in the cytoplasm and the signaling is working, which receives the signal from the upstream HA and membrane Hyal-2. We further showed the in vivo Hyal-2/WWOX complex accumulated in the apoptotic nuclei of neurons in the rat brains during traumatic brain injury. While native HA cannot induce cell death, HA may activate the overly expressed Smad4/Hyal-2/WWOX signaling complex and causes bubbling cell death.

Why does HA enhance cancer metastasis? HA constitutes approximately $20 \%$ of human body weight, and one third of HA undergoes degradation cycle on a daily basis $[57,58]$. Degraded HA long and chain chains, from more than $20000 \mathrm{kDa}$ down to oligomers, may promote cell growth and stimulate angiogenesis [1-8, 59, 60]. In most cases, malignant cancer cells are either devoid of tumor suppressors WWOX, Smad4 and p53 or possess mutations in these proteins, HA induced-signaling of Smad4/Hyal-2/WWOX and Smad4/WWOX/p53 for growth inhibition and death is blocked. This allows cancer cell growth advantage for metastasis [1-8]. Utilization of
A

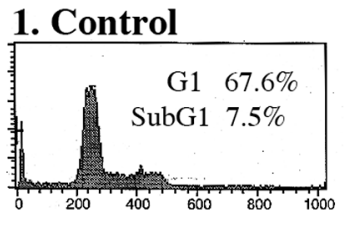

3. Hyal-2 $(2.5 \mu \mathbf{g})$

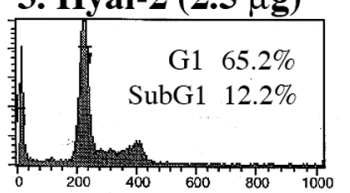

5. Hyal-2 (5 $\mu \mathrm{g})$

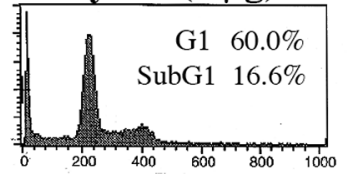

7. Hyal-2 $(10 \mu \mathrm{g})$

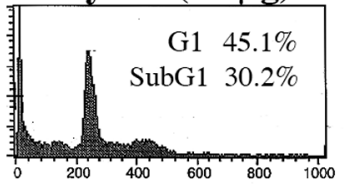

2. Smad4

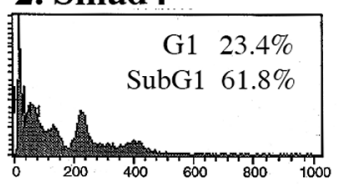

4. Smad4 + Hyal-2 (2.5 $\mu \mathrm{g})$

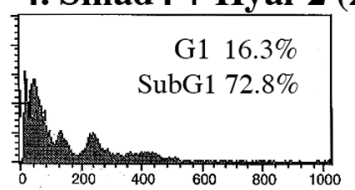

6. Smad4 + Hyal-2 $(5 \mu \mathrm{g})$

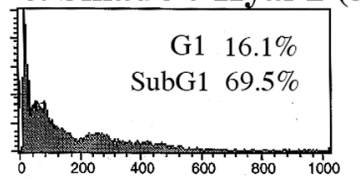

8. Smad4 + Hyal-2 (10 $\mu \mathrm{g})$

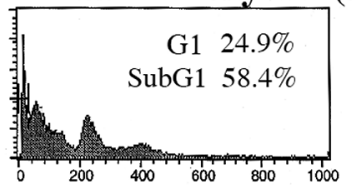

B

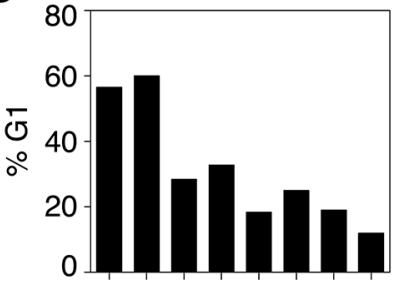

Hyal-2 - + - + + - +

WWOX - - + - +++

Smad4 - - +-+++
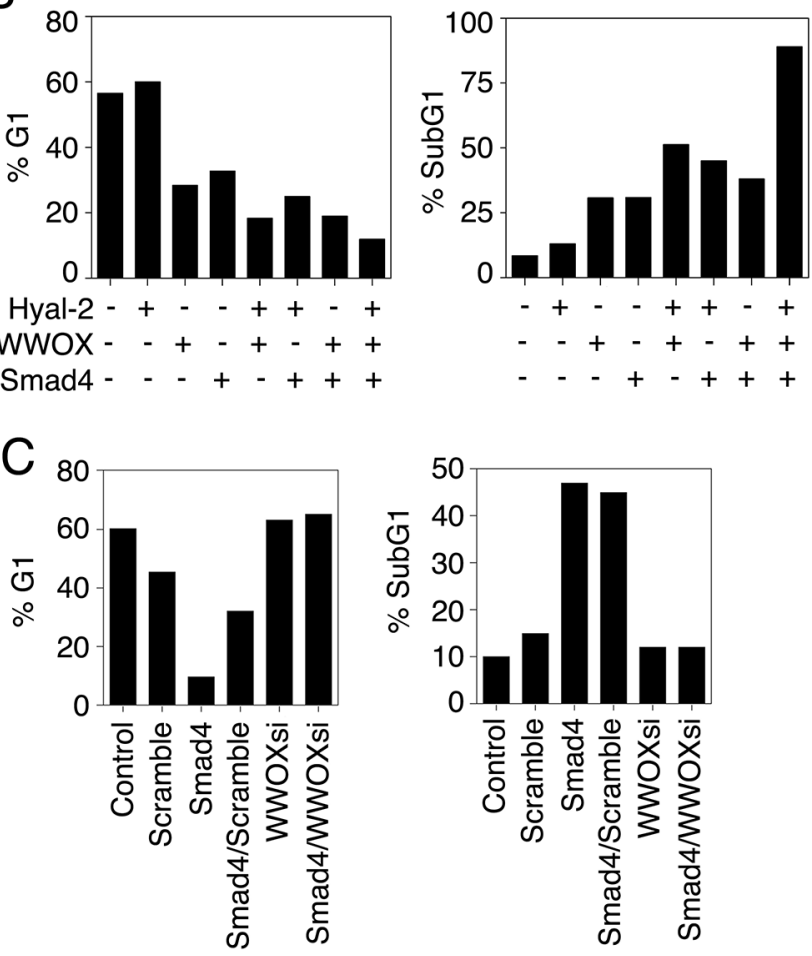

Figure 9: Hyal-2, WWOX and Smad4 synergistically induce apoptosis. A. L929 cells were electroporated with Hyal-2 and/ or Smad4 constructs, cultured $48 \mathrm{hr}$, and subjected to cell cycle analysis by FACS. Hyal-2 could not bind Smad4 (see Figure 5), and did not enhance the apoptotic function of Smad4 (\% apoptosis $=\%$ subG1 phase of the cell cycle). B. Under similar conditions, L929 cells were transfected with Hyal-2, WWOX, and/or Smad4. Hyal-2 enhanced WWOX-mediated apoptosis. In combination, these 3 proteins increased cell death. C. In contrast, WWOXsi blocked Smad4-mediated apoptosis. In controls, scramble siRNA had no effect. WWOXsi, siRNA-targeting WWOX. All data are average from two experiments B, C. Unless otherwise indicated, $5 \mu \mathrm{g}$ DNA constructs were used in electroporation. Data for both G1 and SubG1 phases are shown for all experiments. 
Smad proteins in the HA signaling supports the finding that HA either enhances or inhibits the TGF- $\beta$ signaling $[24,61,62]$. We believe that both HA and TGF- $\beta 1$ could simultaneously compete for utilization of Smads. We showed here that both HA and TGF- $\beta 1$, together, may increase the nuclear localization of Hyal-2/WWOX/Smads complex.

WWOX is essential for neural development [34, 63]. Null mutation of $W W O X / W$ wox gene causes severe neural diseases and early death in humans and animals [22, 34]. HA protects neurons from traumatic brain injuries [64], and this could be related with HA-mediated disappearance of Hyal-2, which reduces the WWOX/Smad4 signaling. During embryonic development, murine WWOX is overexpressed in the cardiac cells and endothelia of blood vessels [63]. Also, high levels of WWOX are shown in the neural crest-derived structures such as cranial and spinal ganglia, skin pigment cells and mesenchyme in the head. The observations are in supporting with those studies in knockout animals and null mutations in humans, and suggest the key role of WWOX in neuronal development $[22,63-65]$.

\section{$3 \mathrm{hr}$ post injury WWOX, $10 \mathrm{~nm}$; Hyal-2, $20 \mathrm{~nm}$}
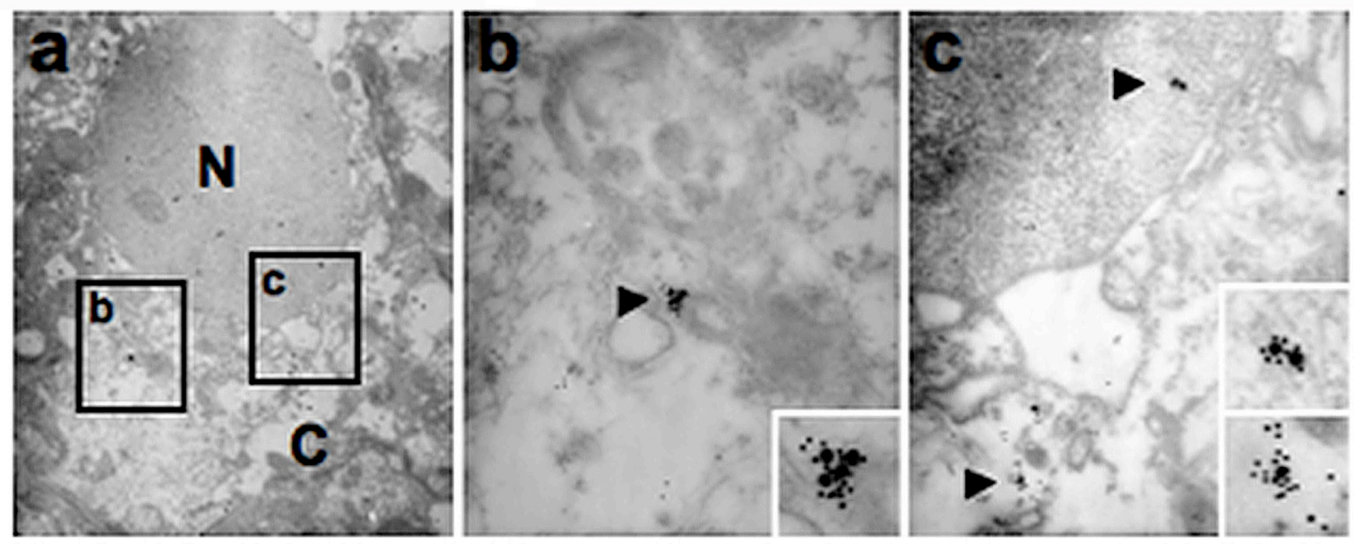

\section{4 hr post injury}
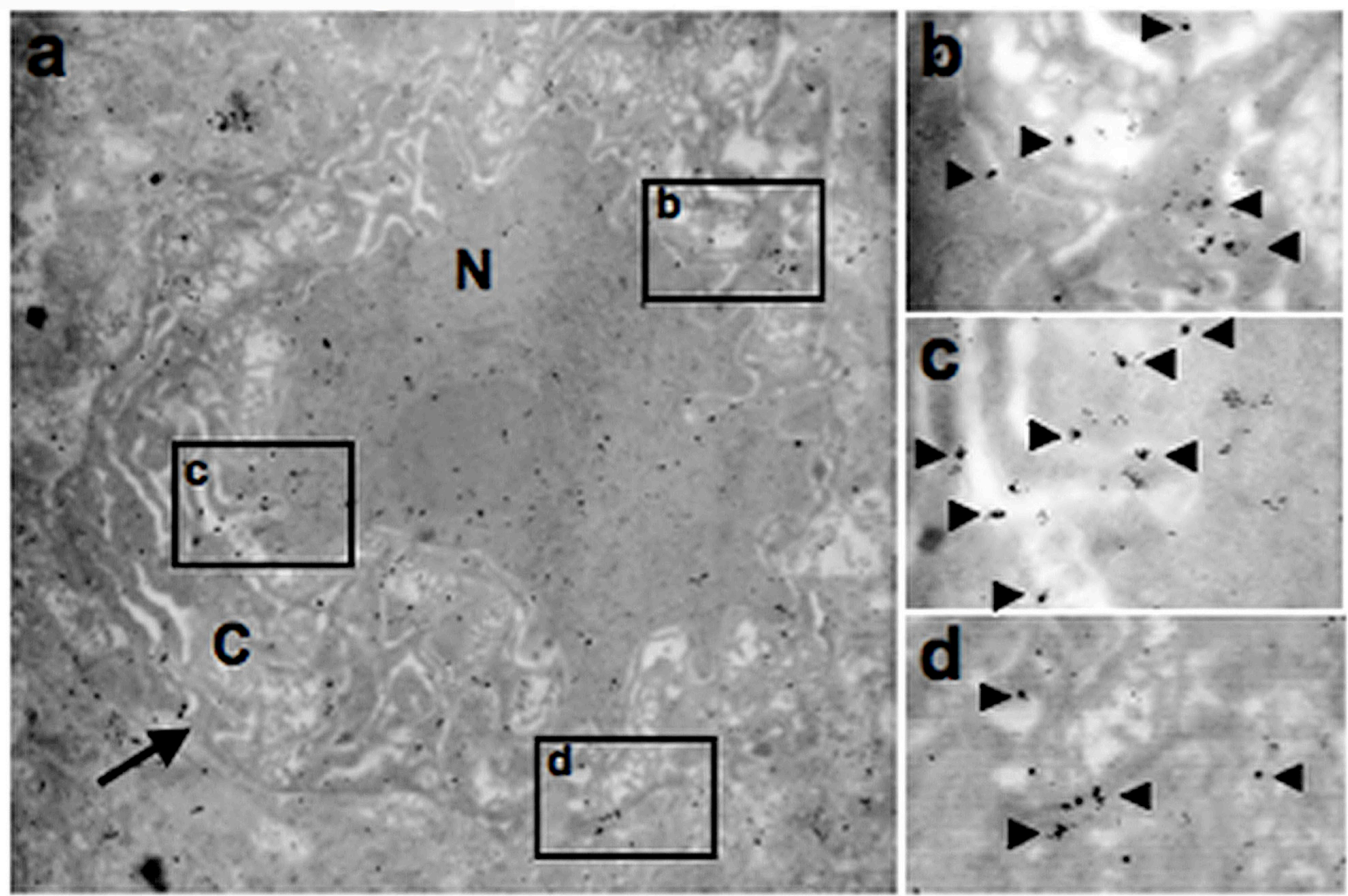

Figure 10: Nuclear accumulation of Hyal-2 and WWOX in apoptotic nuclei of cortical neurons during traumatic brain injury. Rats were stabbed with needles into their brains to induce traumatic brain injury. Post injury for 3 and 24 hr, rats were sacrificed. There were increased numbers of apoptotic neurons in the brain cortex, with the presence of the Hyal-2/WWOX complex in the apoptotic nuclei of neurons. 


\section{MATERIALS AND METHODS}

\section{Cell lines}

The following cell lines were maintained in our laboratory and used for indicated experiments: 1) murine TNF-sensitive L929 and TNF-resistant L929R fibroblasts, 2) human TBRII-deficient colorectal HCT116 cells, 3) human neuroblastoma SK-N-SH cells, 4) human prostate DU145 cells, 5) human breast ER ${ }^{+} \mathrm{WWOX}^{+} \mathrm{MCF}-7$ cells, 6) human breast triple negative MDA-MB-231 and MDAMB-435S cells, 7) human lung p53-deficient NCI-H1299 cells, and 8) monkey kidney COS7, 9) mouse breast cancer 4T1-Luc, 4T1-Luc-c1d, and 4T1-Luc-c5d cells, 10) human monocytic U937 and THP-1 cells, and 11) human T leukemia Jurkat and Molt-4 cells. These cells were from American Type Culture Collections (ATCC), Manassas, VA. 4T1-Luc-c1d, and 4T1-Luc-c5d cells were selected from cold shock challenge at $4{ }^{\circ} \mathrm{C}$ for 1 and 5 days, respectively.

\section{Chemicals, antibodies and western blotting}

Bovine testicular hyaluronidase PH-20, Streptomyces hyalurolyticus hyaluronidase, and human high-molecular-weight umbilical HA were from Sigma-Aldrich. Medical grade HA was from Lifecore Biomedical. $( \pm$ )-Blebbistatin was from Calbiochem. We have generated antibodies against the $N$ - and $C$ termini of WWOX [16, 37, 40], WWOX2 [19], Tyr33phosphorylated WWOX [37], GST-WWOX [37], and Hyal-2 [21]. Commercial antibodies used were against: WWOX (N-19), JNK1, p53 (full length), Smad2, Smad3, Smad4, phospho-Smad2/3 (at Ser433/435), Tyr204phosphorylated ERK (p-ERK) (the above antibodies from Santa Cruz Laboratories), phospho-Smad2 (Calbiochem), pS15-p53 (Calbiochem), pS46-p53 (R\&D Systems), phospho-FAK (Upstate Biotechnology), and $\alpha$-tubulin (Accurate Chemicals). Where indicated, Western blottings were carried out by our standard procedures $[16,17,21$, 23, 24, 23-25].

\section{Immunofluorescence microscopy and immunoelectron microscopy}

DU145, COS7 and other indicated cells were cultured $48 \mathrm{hr}$ on cover slides, fixed with formaldehyde (3.3\%), permeabilized with $0.5 \%$ Triton-X100 (in the formaldehyde solution), and then stained with indicated primary antibodies and secondary fluorescent antibodies, followed by processing fluorescence microscopy $[16,19,21,23,50]$. Nuclei were stained with DAPI (4',6-diamidino-2-phenylindole) (Calbiochem). Where indicated, immunoelectron microscopy was carried out as described [21, 40, 51]. Briefly, HCT116 cells were fixed for 1 hour using a freshly prepared $4 \%$ solution of paraformaldehyde in phosphate-buffered saline, immersed in $1 \%$ osmium tetroxide, dehydrated in a graded series of ethanol, and finally embedded in resin (EMS, SPUR's Kit). Ultrathin sections $(70-80 \mathrm{~nm})$ were prepared with an ultramicrotome (Reichert-Jung) and hybridized with an aliquot of IgG antibody against WWOX, p-WWOX, Smad4, or Hyal-2, followed by probing with a secondary anti-rabbit IgG $20 \mathrm{~nm}$ A-gold probe or anti-mouse (or goat) $10 \mathrm{~nm}$ gold particles. The sections were stained with saturated aqueous uranyl acetate and lead citrate at room temperature. Specimens were then observed under a transmission electron microscopy (JEOL JEM-1200EX, Japan) at $100 \mathrm{kV}$.

\section{cDNA expression constructs and transfection in cell lines and co-immunoprecipitation}

The following expression constructs were made: 1) murine EGFP-WWOX [16], and 2) murine dominantnegative full length WWOX (dn-WWOX) and the $N$ terminal WW domain of WWOX (dn-WW), tagged with EGFP [16, 37]. The mutations were K28T and D29V. Murine Smad4 cDNA (from ATCC), encoding a truncated 43-kDa protein (GenBank accession AY493561) was tagged with in-frame with the $C$-terminal EGFP [21]. A plasmid construct was made in pSuppressorNeo vector for expressing siRNA to inhibit the expression of human and mouse WWOX protein [37].

Where indicated, COS7 or other cells were electroporated with the above constructs (200 volt, 50 msec; Square Wave BTX ECM830, Genetronics), cultured overnight, and then treated with hyaluronan and/or bovine hyaluronidase PH-20 (Sigma Chemicals) for indicated times, followed by preparing cytosolic and nuclear fractions [16, 21, 23, 37, 39, 41, 42]. The extent of protein expression was determined using specific antibodies. Coimmunoprecipitation was performed as described [16, 21, 23, 37, 39, 41, 42]. Briefly, SK-N-SH and other indicated cell lines were cultured overnight (in $150 \mathrm{~mm}$ Petri dishes) and then treated with HA $(25 \mu \mathrm{g} / \mathrm{ml})$ for $30 \mathrm{~min}$, followed by processing immunoprecipitation using cytosolic and nuclear fractions. Where indicated, non-immune serum or $\mathrm{IgG}$ was used in negative controls.

\section{Cytoplasmic yeast two-hybrid analysis}

Ras rescue-based yeast two-hybrid analysis (CytoTrap; Stratagene) was performed [16, 21, 37, 39, 42]. Briefly, activation of Ras signaling pathway in yeast occurs as a result of binding of a cytosolic Sos-tagged bait protein to a cell membrane-anchored target protein (tagged with a myristoylation signal). This activation allows mutant yeast $\operatorname{cdc} 25 \mathrm{H}$ to grow in $37^{\circ} \mathrm{C}$ using a selective agarose plate containing galactose. Without binding, yeast cells fail to grow at $37^{\circ} \mathrm{C}$. Target constructs made in a 
pMyr vector (with the myristoylation signal) were murine Smad4, murine Hyal-2, and human p53. Bait constructs made in a pSos vector (tagged with an $N$-terminal Sos protein) were murine WWOX, WWOXww (the first WW domain), WWOXww(Y33R), WWOXsdr (the entire SDR domain), WWOXsdr(mito) (the mitochondria-targeting region in the SDR domain), and WWOX(K28T/D29V) [16, 21, 37]. Additionally, constructs for a self-binding positive control were MafB (in both pMyr and pSos).

\section{Time lapse tri-molecular FRET microscopy}

FRET microscopy was carried out [21, 23, 42]. DU145 cells were transiently overexpressed with each set of the following constructs: 1) ECFP-Smad4, EGFP-WWOX, DsRed-monomer-p53; 2) ECFP-Smad4, EGFP-Hyal-2(-sp), DsRed-monomer-WWOX; 3) ECFP-I $\kappa \mathrm{B} \alpha$, EGFP-ERK, DsRed-monomer-WWOX [42]. Where indicated dominant negative WWOX(K28T/D29V) or p53(S46G) constructs was used to replace the wild type. FRET microscopy was performed using an inverted fluorescence microscope (Nikon Eclipse TE-2000U) and the data were analyzed as described.

\section{Image analysis and data presentation}

We analyzed images by the NIH Image and Photoshop softwares. All experiments in this study were repeated 2-5 times.

\section{ACKNOWLEDGMENTS}

This work was supported, in part, by the Guthrie Foundation for Education and Research, the American Heart Association, the Department of Defense USA (W81XWH-08-1-0682), and the Ministry of Science and Technology, Taiwan, ROC (105-2320-B-006-046 and 1052320-B-006-036). This article is in our memory of Professor Shur-Tzu Chen with deepest respect and great love. Dr. Chen passed away on February 28, 2015 with lymphoma.

\section{CONFLICTS OF INTEREST}

The authors declare no conflicts of interest.

\section{Authors' contributions}

LS, JH, DZL, ZLL, SRL and MHL: carried out cloning experiments, SDS-PAGE, and Western blottings; $\mathrm{LJH}$ and QH: carried out yeast two-hybrid analysis; STC: carried out traumatic brain injury experiments by immunoelectron microscopy; HLK: Time lapse FRET microscopy and immunofluorescence microscopy; HCC: immunofluorescence microscopy; GA: provided medical grade hyaluronan and discussion on the paper; $\mathrm{LJH}$ and NSC: proofread and discussed the manuscript; NSC: wrote the manuscript, conceived ideas, designed experiments and performed co-immunoprecipitation and yeast twohybrid analysis, and immunofluorescence microscopy.

\section{REFERENCES}

1. Stern R. Devising a pathway for hyaluronan catabolism: are we there yet? Glycobiology. 2003; 13:105r-15r.

2. Sherman LS, Matsumoto S, Su W, Srivastava T, and Back SA. Hyaluronan Synthesis, Catabolism, and Signaling in Neurodegenerative Diseases. Int J Cell Biol. 2015; 2015:368584. Review.

3. Schmaus A, Bauer J, and Sleeman JP. Sugars in the microenvironment: the sticky problem of HA turnover in tumors. Cancer Metastasis Rev. 2014; 33:1059-79.

4. McAtee CO, Barycki JJ, and Simpson MA. Emerging roles for hyaluronidase in cancer metastasis and therapy. Adv Cancer Res. 2014; 123:1-34.

5. Frost GI, Mohapatra G, Wong TM, Csoka AB, Gray JW, and Stern R. HYAL1(LUCA-1), a candidate tumor suppressor gene on chromosome 3 p21.3, is inactivated in head and neck squamous cell carcinomas by aberrant splicing of premRNA. Oncogene. 2000; 19:870-7.

6. Franzmann EJ, Schroeder GL, Goodwin WJ, Weed DT, Fisher P, and Lokeshwar VB. Expression of tumor markers hyaluronic acid and hyaluronidase (HYAL1) in head and neck tumors. Int J Cancer. 2003; 106:438-45.

7. Kultti A, Li X, Jiang P, Thompson CB, Frost GI, and Shepard HM. Therapeutic targeting of hyaluronan in the tumor stroma. Cancers (Basel). 2012; 4:873-903.

8. Csoka TB, Frost GI, Heng HHQ, Scherer SW, Mohapatra $\mathrm{G}$, and Stern R. The hyaluronidase gene HYAL1 maps to chromosome 3p21.2-p21.3 in human and 9F1-F2 in mouse, a conserved candidate tumor suppressor locus. Genomics. 1998; 48:63-70.

9. Lepperdinger G, Strobl B, and Kreil G. HYAL2, a human gene expressed in many cells, encodes a lysosomal hyaluronidase with a novel type of specificity. J Biol Chem. 1998; 273:22466-70.

10. Rai SK, Duh FM, Vigdorovich V, Danilkovitch-Miagkova A, Lerman MI, and Miller AD. Candidate tumor suppressor HYAL2 is a glycosylphosphatidylinositol (GPI)-anchored cell-surface receptor for jaagsiekte sheep retrovirus, the envelope protein of which mediates oncogenic transformation. Proc Natl Acad Sci USA. 2001; 98:4443-8.

11. Duterme C, Mertens-Strijthagen J, Tammi M, and Flamion B. Two novel functions of hyaluronidase-2 (Hyal2) are formation of the glycocalyx and control of CD44-ERM interactions. J Biol Chem. 2009; 284:33495-508.

12. Bourguignon LYW, Singleton PA, Diedrich F, Stern R, and Gilad E. CD44 interaction with $\mathrm{Na}+\mathrm{H}+$ exchanger (NHE1) creates acidic microenvironments leading to hyaluronidase-2 and cathepsin B activation and breast tumor cell invasion. J Biol Chem. 2004; 279:26991-7007. 
13. Beech DJ, Madan AK, and Deng N. Expression of PH-20 in normal and neoplastic breast tissue. J Surg Res. 2002; 103:203-7.

14. Chang NS. Hyaluronidase enhancement of TNF-mediated cell death is reversed by TGF-beta 1. Am J Physiol-Cell Ph. 1997; 273:C1987-94.

15. Chang NS, Carey G, Pratt N, Chu E, and Ou M. p53 overexpression and downregulation of inter-alpha-inhibitor are associated with hyaluronidase enhancement of TNF cytotoxicity in L929 fibroblasts. Cancer Lett. 1998; 131:45-54.

16. Chang NS, Pratt N, Heath J, Schultz L, Sleve D, Carey GB, and Zevotek N. Hyaluronidase induction of a WW domaincontaining oxidoreductase that enhances tumor necrosis factor cytotoxicity. J Biol Chem. 2001; 276:3361-70.

17. Chang NS, Hsu LJ, Lin YS, Lai FJ, and Sheu HM. WW domain-containing oxidoreductase: a candidate tumor suppressor. Trends Mol Med. 2007; 13:12-22.

18. Chang JY, He RY, Lin HP, Hsu LJ, Lai FJ, Hong Q, Chen SJ, and Chang NS. Signaling from membrane receptors to tumor suppressor WW domain-containing oxidoreductase. Exp Biol Med (Maywood). 2010; 235:796-804. Review.

19. Sze CI, Su M, Pugazhenthi S, Jambal P, Hsu LJ, Heath J, Schultz L, and Chang NS. Down-regulation of WW domaincontaining oxidoreductase induces Tau phosphorylation in vitro. A potential role in Alzheimer's disease. J Biol Chem. 2004; 279:30498-506.

20. Nikitovic D, Kouvidi K, Karamanos NK, and Tzanakakis GN. The roles of hyaluronan/RHAMM/CD44 and their respective interactions along the insidious pathways of fibrosarcoma progression. Biomed Res Int. 2013; 2013:929531.

21. Hsu LJ, Schultz L, Hong Q, Van Moer K, Heath J, Li MY, Lai FJ, Lin SR, Lee MH, Lo CP, Lin YS, Chen ST, and Chang NS. Transforming growth factor betal signaling via interaction with cell surface Hyal-2 and recruitment of WWOX/WOX1. J Biol Chem. 2009; 284:16049-59.

22. Chang NS. Introduction to a thematic issue for WWOX. Exp Biol Med (Maywood). 2015; 240:281-4.

23. Lee MH, Su WP, Wang WJ, Lin SR, Lu CY, Chen YA, Chang JY, Huang SS, Chou PY, Ye SR, Chen SJ, He H, Liu TH, et al. Zfra activates memory Hyal-2+ CD3CD19- spleen cells to block cancer growth, stemness, and metastasis in vivo. Oncotarget. 2015; 6:3737-3751. doi: 10.18632/oncotarget.2895.

24. Chang NS. Transforming growth factor-beta 1 blocks the enhancement of tumor necrosis factor cytotoxicity by hyaluronidase Hyal-2 in L929 fibroblasts. BMC Cell Biol. 2002; 3:8.

25. Bednarek AK, Laflin KJ, Daniel RL, Liao QY, Hawkins KA, and Aldaz CM. WWOX, a novel WW domain-containing protein mapping to human chromosome 16q23.3-24.1, a region frequently affected in breast cancer. Cancer Res. 2000; 60:2140-5.
26. Ried K, Finnis M, Hobson L, Mangelsdorf M, Dayan S, Nancarrow JK, Woollatt E, Kremmidiotis G, Gardner A, Venter D, Baker E, and Richards RI. Common chromosomal fragile site FRA16D sequence: identification of the FOR gene spanning FRA16D and homozygous deletions and translocation breakpoints in cancer cells. Hum Mol Genet. 2000; 9:1651-63.

27. Abu-Remaileh M, Joy-Dodson E, Schueler-Furman O, and Aqeilan RI. Pleiotropic Functions of Tumor Suppressor WWOX in Normal and Cancer Cells. J Biol Chem. 2015; 290:30728-35.

28. Del Mare S, Husanie H, Iancu O, Abu-Odeh M, Evangelou K, Lovat F, Volinia S, Gordon J, Amir G, Stein JL, Stein GS, Croce CM, Gorgoulis VG, et al. p53 and WWOX dysregulation synergize to drive the development of osteosarcoma. Cancer Res. 2016; in press.

29. Lo JY, Chou YT, Lai FJ, and Hsu LJ. Regulation of cell signaling and apoptosis by tumor suppressor WWOX. Exp Biol Med (Maywood). 2015; 240:383-91.

30. Schrock MS, and Huebner K. WWOX: a fragile tumor suppressor. Exp Biol Med (Maywood). 2015; 240:296-304.

31. Richards RI, Choo A, Lee CS, Dayan S, and O'Keefe L. WWOX, the chromosomal fragile site FRA16D spanning gene: its role in metabolism and contribution to cancer. Exp Biol Med (Maywood). 2015; 240:338-44.

32. O'Keefe LV, Lee CS, Choo A, and Richards RI. Tumor Suppressor WWOX Contributes to the Elimination of Tumorigenic Cells in Drosophila melanogaster. PLoS One. 2015; 10:e0136356.

33. Tsai CW, Lai FJ, Sheu HM, Lin YS, Chang TH, Jan MS, Chen SM, Hsu PC, Huang TT, Huang TC, Sheen MC, Chen ST, Chang WC, et al. WWOX suppresses autophagy for inducing apoptosis in methotrexate-treated human squamous cell carcinoma. Cell Death Dis. 2013; 4:e792.

34. Chang HT, Liu CC, Chen ST, Yap YV, Chang NS, and Sze CI. WW domain-containing oxidoreductase in neuronal injury and neurological diseases. Oncotarget 2014; 5:117929. doi: 10.18632/oncotarget.2961.

35. Alkhateeb AM, Aburahma SK, Habbab W, and Thompson IR. Novel mutations in WWOX, RARS2, and C10orf2 genes in consanguineous Arab families with intellectual disability. Metab Brain Dis. 2016; 31:901-7.

36. Aldaz CM, Ferguson BW, Abba MC. WWOX at the crossroads of cancer, metabolic syndrome related traits and CNS pathologies. Biochim Biophys Acta. 2014; 1846:188-200.

37. Chang NS, Doherty J, and Ensign A. JNK1 physically interacts with WW domain-containing oxidoreductase (WOX1) and inhibits WOX1-mediated apoptosis. J Biol Chem. 2003; 278:9195-202.

38. Lai FJ, Cheng CL, Chen ST, Wu CH, Hsu LJ, Lee JY, Chao SC, Sheen MC, Shen CL, Chang NS, and Sheu HM. WOX1 is essential for UVB irradiation-induced apoptosis and down-regulated via translational blockade in UVB-induced 
cutaneous squamous cell carcinoma in vivo. Clin Cancer Res. 2005; 11:5769-77.

39. Chang NS, Doherty J, Ensign A, Schultz L, Hsu LJ, and Hong Q. WOX1 is essential for TNF-, UV light-, staurosporine-, and p53-mediated cell death and its tyrosine 33 phosphorylated form binds and stabilizes serine 46-phosphorylated p53. J Biol Chem. 2005; 280:43100-8.

40. Chen ST, Chuang JI, Cheng CL, Hsu LJ, and Chang NS. Light-induced retinal damage involves tyrosine 33 phosphorylation, mitochondrial and nuclear translocation of WW domain-containing oxidoreductase in vivo. Neuroscience. 2005; 130:397-407.

41. Chang NS, Schultz L, Hsu LJ, Lewis J, Su M, and Sze CI. 17 beta-Estradiol upregulates and activates WOX1/ WWOXv1 and WOX2/WWOXv2 in vitro: potential role in cancerous progression of breast and prostate to a premetastatic state in vivo. Oncogene. 2005; 24:714-23.

42. Huang SS, Su WP, Lin HP, Kuo HL, Wei HL, and Chang NS. Role of WW domain-containing oxidoreductase WWOX in Driving T Cell Acute Lymphoblastic Leukemia Maturation. J Biol Chem. 2016; 291:17319-31.

43. Jin C, Ge L, Ding X, Chen Y, Zhu H, Ward T, Wu F, Cao X, Wang Q, and Yao X. PKA-mediated protein phosphorylation regulates ezrin-WWOX interaction. Biochem Biophys Res Commun. 2006; 341:784-91.

44. Bourguignon LYW, Singleton PA, Zhu HB, and Zhou B. Hyaluronan promotes signaling interaction between CD44 and the transforming growth factor beta receptor I in metastatic breast tumor cells. J Biol Chem. 2002; 277:39703-12.

45. Wollin M, Fitzgerald TJ, Santucci MA, Menon M, Longcope C, Reale F, Carlson J, Sakakeeny MA, and Greenberger JS. Radiosensitivity of human-prostate cancer and malignant-melanoma cell-lines. Radiother Oncol. 1989; 15:285-93.

46. Park BJ, Park JI, Byun DS, Park JH, and Chi SG. Mitogenic conversion of transforming growth factor-beta 1 effect by oncogenic Ha-Ras-induced activation of the mitogenactivated protein kinase signaling pathway in human prostate cancer. Cancer Res. 2000; 60:3031-8.

47. Isaacs WB, Carter BS, and Ewing CM. Wild-type p53 suppresses growth of human prostate-cancer cells containing mutant p53 alleles. Cancer Res. 1991; 51:4716-20.

48. Coezy E, Borgna JL, and Rochefort H. Tamoxifen and metabolites in MCF7 cells - correlation between binding to estrogen-receptor and inhibition of cell growth. Cancer Res. 1982; 42:317-23.

49. Ferguson BW, Gao X, Zelazowski MJ, Lee J, Jeter CR, Abba MC, and Aldaz CM. The cancer gene WWOX behaves as an inhibitor of SMAD3 transcriptional activity via direct binding. BMC Cancer. 2013; 13:593.

50. Lin HP, Chang JY, Lin SR, Lee MH, Huang SS, Hsu LJ, and Chang NS. Identification of an In Vivo MEK/WOX1
Complex as a Master Switch for Apoptosis in T Cell Leukemia. Genes Cancer. 2011; 2:550-62.

51. Chen SJ, Lin PW, Lin HP, Huang SS, Lai FJ, Sheu HM, Hsu LJ, and Chang NS. UV irradiation/cold shockmediated apoptosis is switched to bubbling cell death at low temperatures. Oncotarget. 2015; 6:8007-18. doi: 10.18632/ oncotarget.3153.

52. Chang NS. Bubbling cell death: A hot air balloon released from the nucleus in the cold. Exp Biol Med (Maywood). 2016; 241:1306-15.

53. Otsuka N, Tomonaga M, and Ikeda K. Rapid appearance of beta-amyloid precursor protein immunoreactivity in damaged axons and reactive glial cells in rat brain following needle stab injury. Brain Res. 1991; 568:335-8.

54. Chang JY, and Chang NS. WWOX dysfunction induces sequential aggregation of TRAPPC6A $\Delta$, TIAF1, tau and amyloid $\beta$, and causes apoptosis. Cell Death Discov. 2015; 1:15003.

55. Chang JY, Lee MH, Lin SR, Yang LY, Sun HS, Sze CI, Hong Q, Lin YS, Chou YT, Hsu LJ, Jan MS, Gong CX, and Chang NS. Trafficking protein particle complex 6A delta (TRAPPC6A $\triangle$ ) is an extracellular plaque-forming protein in the brain. Oncotarget. 2015; 6:3578-89. doi: 10.18632/ oncotarget.2876.

56. Sze CI, Kuo YM, Hsu LJ, Fu TF, Chiang MF, Chang JY, and Chang NS. A cascade of protein aggregation bombards mitochondria for neurodegeneration and apoptosis under WWOX deficiency. Cell Death Dis. 2015; 6:e1881.

57. Lee JY, and Spicer AP. Hyaluronan: a multifunctional, megaDalton, stealth molecule. Curr Opin Cell Biol. 2000; 12:581-6.

58. Stern R. Hyaluronan catabolism: a new metabolic pathway. Eur J Cell Biol. 2004; 83:317-25.

59. Slevin M, Krupinski J, Kumar S, and Gaffney J. Angiogenic oligosaccharides of hyaluronan induce protein tyrosine kinase activity in endothelial cells and activate a cytoplasmic signal transduction pathway resulting in proliferation. Lab Invest. 1998; 78:987-1003.

60. Slevin M, Kumar S, and Gaffney J. Angiogenic oligosaccharides of hyaluronan induce multiple signaling pathways affecting vascular endothelial cell mitogenic and wound healing responses. J Biol Chem. 2002; 277:41046-59.

61. Ito T, Williams JD, Fraser D, and Phillips AO. Hyaluronan attenuates transforming growth factor-beta 1-mediated signaling in renal proximal tubular epithelial cells. Am J Pathol. 2004; 164:1979-88.

62. Ito T, Williams JD, Fraser DJ, and Phillips AO. Hyaluronan regulates transforming growth factor-beta 1 receptor compartmentalization. J Biol Chem. 2004; 279:25326-32.

63. Chen ST, Chuang JI, Wang JP, Tsai MS, Li H, and Chang NS. Expression of WW domain-containing oxidoreductase WOX1 in the developing murine nervous system. Neuroscience. 2004; 124:831-9. 
64. Wang J, Wang X, Wei J, and Wang M. Hyaluronan tetrasaccharide exerts neuroprotective effect and promotes functional recovery after acute spinal cord injury in rats. Neurochem Res. 2015; 40:98-108.

65. Li MY, Lai FJ, Hsu LJ, Lo CP, Cheng CL, Lin SR, Lee MH, Chang JY, Subhan D, Tsai MS, Sze CI, Pugazhenthi
S, Chang NS, and Chen ST. Dramatic co-activation of WWOX/WOX1 with CREB and NF-kappaB in delayed loss of small dorsal root ganglion neurons upon sciatic nerve transection in rats. PLoS One. 2009; 4:e7820. 